\title{
GROUPS OF PIECEWISE LINEAR HOMEOMORPHISMS
}

\author{
MELANIE STEIN
}

\begin{abstract}
In this paper we study a class of groups which may be described as groups of piecewise linear bijections of a circle or of compact intervals of the real line. We use the action of these groups on simplicial complexes to obtain homological and combinatorial information about them. We also identify large simple subgroups in all of them, providing examples of finitely presented infinite simple groups.
\end{abstract}

\section{INTRODUCTION}

The first example of a finitely presented infinite simple group was discovered in 1965 by $\mathrm{R}$. J. Thompson. This group, $G$, arose in the study of logic, but has several other descriptions. One of them is as the automorphism group of a certain algebraic object, the Jónsson-Tarski algebra. In 1974 G. Higman [9], using this description, generalized $G$ to an infinite family of groups called $G_{n, r}$ (in Higman's notation $G=G_{2,1}$ ) . $G$ has another description as a group of homeomorphisms of the Cantor set. It has subgroups, also studied by Thompson, which may be described as homeomorphism groups of the circle and the unit interval. Using the interpretation as homeomorphism groups, Robert Bieri and Ralph Strebel, in a set of unpublished notes, considered more general homeomorphism groups of the real line.

In this paper we will consider a class of groups containing Higman's groups as well as the groups of Bieri and Strebel which are supported on compact intervals. We obtain homological and combinatorial information about them, and extend the known simplicity results.

To define the groups, let $P$ be a multiplicative subgroup of the positive real numbers, and let $A$ be a $\mathbb{Z} P$-submodule of the reals with $P \cdot A=A$. Choose a number $l \in A, l>0$. Let $F(l, A, P)$ be the group of piecewise linear homeomorphisms of $[0, l]$ with finitely many singularities, all in $A$, having slopes only in $P$. Similarly, we can define a larger group $T(l, A, P)$ to be the group of piecewise linear homeomorphims of $[0, l] /_{\{0, l\}}$ (the circle formed by identifying the endpoints of the closed interval $[0, l]$ ) with finitely many singularities in $A$ and slopes in $P$, with the additional requirement that the homeomorphisms send $A \cap[0, l]$ to itself. Finally, we define $G(l, A, P)$ to be the group of right continuous bijections of $[0, l)$ which are piecewise linear, with finitely many

Received by the editors May 12, 1990.

1980 Mathematics Subject Classification (1985 Revision). Primary 20J05; Secondary 20 E32. 
discontinuities and singularities, all in $A$, slopes in $P$, and mapping $A \cap[0, l)$ to itself. Then we have $F(l, A, P) \subset T(l, A, P) \subset G(l, A, P)$. To relate these to the groups in the literature, we remark that $G\left(1, \mathbb{Z}\left[\frac{1}{2}\right],\langle 2\rangle\right)$ is Thompson's finitely presented infinite simple group, and the $T$ and $F$ subgroups are the subgroups studied by Thompson. Also, $G\left(r, \mathbb{Z}\left[\frac{1}{n}\right],\langle n\rangle\right)$, where $r, n \in \mathbb{Z}$, is Higman's group $G_{n, r}$.

In [2], K. S. Brown uses the action of $G\left(r, \mathbb{Z}\left[\frac{1}{n}\right],\langle n\rangle\right)$ and the corresponding $F$ and $T$ subgroups on simplicial complexes to show that they are all finitely presented and of type $F P_{\infty}$. He then generalizes the complexes to ones on which $G\left(r, \mathbb{Z}\left[\frac{1}{n_{1} \cdots n_{k}}\right],\left\langle n_{1}, \ldots, n_{k}\right\rangle\right)$ and the $F$ and $T$ subgroups act, where $n_{1}, \ldots, n_{k}$ and $r$ are positive integers (unpublished). In $\S 1$ we will describe these complexes (we will call them $X$ ) and find inside them homotopy equivalent subcomplexes (called $N$ ) on which the groups also act. This yields nice infinite presentations for our groups.

In $\S 2$ we restrict ourselves to the $F$ groups with $P$ free abelian of finite rank, still generated by integers. We collapse the quotient complexes $N / F$ to complexes with only finitely many cells in each dimension. This shows the groups to be of type $F P_{\infty}$ and finitely presented. In the rank one case, this collapse also easily yields the homology of $F$.

In $\S 3$ we construct a slightly different complex $K$, homotopy equivalent to $N$. We then describe how to more completely collapse the quotient complex $K / F$ to a homotopy equivalent complex with finitely many cells in each dimension. This provides smaller finite presentations for the groups, and easily gives the homology of $F$ in the rank one case and some of the rank two examples.

In $\S 4$ we consider the cases of slope group of rank one or two, and compute the homology of these groups algebraically, using a ring structure with which the chain complexes are equipped.

Recall that Thompson's group was of interest because it was simple and finitely presented. In [9], Higman shows that his generalizations of Thompson's group always have simple commutator subgroups. In [2], Brown extends this result by showing that the corresponding $T$ subgroups have simple second commutator subgroups, and the $F$ subgroups have simple commutator subgroups. Furthermore, Bieri and Strebel observe (see the Appendix) that for any choice of $l, A$, and $P$, the subgroup of $F(l, A, P)$ consisting of homeomorphisms supported on some proper open subinterval of $(0, l)$ has a simple commutator subgroup. It follows from 4.1 that this subgroup is just the commutator subgroup of $F$, so the general $F$ group has simple commutator subgroup. In $\S 5$ we complete this picture by showing that the general $G$ group has a simple commutator subgroup and the general $T$ group has a simple second commutator subgroup.

In [9], Higman also computes the abelianizations of his groups and finds that the commutator subgroups have index 1 or 2 . In $\S 6$ we compute abelianizations for the $G$ groups with $P$ generated by a finite set of integers, using the action of $G$ on the complex $N$. It turns out to be finite, showing that all of the commutator subgroups are finitely presented infinite simple groups. We note here that E. Scott has generalized the groups of Higman in another way to obtain another set of examples of finitely presented infinite simple groups (see $[12,13$, 14]). 
We include an Appendix presenting some unpublished results of Bieri and Strebel. A.1, A.2, and A.3 are all used in $\S 1$, and it might be helpful to read them before beginning $\S 1$. A.4 is needed in $\S 4$, and A.5 is a simplicity result which is not needed in this paper, but is referred to above.

I am very grateful to Ken Brown for giving generous support and advice during the preparation of this work.

\section{THE COMPLEXES}

In this section we will describe the complexes of $\mathrm{K}$. S. Brown on which the $F, T$, and $G$ groups act in the case where $P$ is generated by the set of integers $\left\{n_{1}, n_{2}, \ldots, n_{k}\right\}$ and $A=\mathbb{Z}\left[\frac{1}{n_{1}}, \frac{1}{n_{2}}, \ldots, \frac{1}{n_{k}}\right]$. These complexes are described in [2] for the case where $P$ is generated by one integer; the generalization to more than one integer is unpublished. We will then find a homotopy equivalent $G$ (or $F$ or $T$ ) invariant subcomplex which we will use in computations. We remind the reader that it might be helpful to look at A.1, A.2, and A.3 of the Appendix before beginning this section.

The complexes are constructed assuming that $\left\{n_{1}, \ldots, n_{k}\right\}$ form a basis for $P$, and that $l \in \mathbb{Z}^{+}$. Before describing the complexes we explain why there is no loss of generality in making these assumptions. First, we have

Proposition 1.1 (K. S. Brown). P has a basis as a free abelian group consisting of integers.

Proof of 1.1. Think of $\mathbf{Q}_{>0}$ as a free abelian group with the set of primes as basis. Written additively, $\mathbf{Q}_{>0} \cong L=\mathbb{Z} \oplus \mathbb{Z} \oplus \cdots$ and $P \cong W$, a finitely generated subgroup of $L$ whose generators have only nonnegative coordinates. Choose a maximal independent subset $B^{\prime}$ of the original generating set for $W$, and let $W^{\prime}$ be the group generated by $B^{\prime}$. Then $W^{\prime}$ has finite index in $W$, so $m W \subseteq W^{\prime}$ for some positive integer $m$. But if we choose any basis for $m W$ and let $M$ be the matrix whose rows express the basis for $m W$ in terms of $B^{\prime}$, then performing row operations on $M$ just corresponds to changing the basis for $m W$. We can do enough of these to make all entries below the diagonal zero, and then we can make the remaining entries positive. So $m W$ has a new basis $B$ consisting of $\mathbb{Z}_{>0}$-linear combinations of the elements of $B^{\prime}$. But any element of this basis has all coordinates divisible by $m$, so $\frac{1}{m} B$ is a basis for $W$, consisting of elements which have all coordinates nonnegative, which means that it corresponds to an integral basis for $P$.

Now we consider the choice of the interval on which the homeomorphisms act. Since $l \in A, l$ can be written as $\frac{m}{n}$, where $m, n \in \mathbb{Z}$ and $n \in P$. Then if $\varphi$ is the homeomorphism taking $[0, m]$ to $[0, l]$ with constant slope $\frac{1}{n}$, conjugation by $\varphi$ gives an isomorphism between $G(l, A, P)$ and $G(m, A, P)$ (similarly for $F$ and $T$ ). So we need only consider values of $l \in \mathbb{Z}^{+}$.

From now on we may assume that $\left\{n_{1}, n_{2}, \ldots, n_{k}\right\}, n_{i} \in \mathbb{Z}$, form a basis for $P$ and that $l \in \mathbb{Z}^{+}$. In this situation Brown gives a poset on which the groups act. Here we will first describe everything for the $F$ groups, and then indicate the changes necessary for the $T$ and $G$ groups. We first set $d=$ 
$\operatorname{gcd}\left(n_{1}-1, n_{2}-1, \ldots, n_{k}-1\right)$. An element of the poset is a piecewise linear homeomorphism from $[0, a]$ to $[0, l]$, where $a \in \mathbb{Z}, a \equiv l(\bmod d)$, which has finitely many singularities, all in $A$, and slopes in $P$. To explain where the mod $d$ condition comes from, recall from A.1 that a P.L. homeomorphism with slopes in $P$ and singularities in $A$ can take $[0, a]$ to $[0, l]$ if and only if $a-l \in$ $I P \cdot A$. But $A / I P \cdot A \simeq \mathbb{Z} / d \mathbb{Z}$ in our situation, so we can have such a map if and only if $a \equiv l(\bmod d)$. To make these maps into a poset, given $f_{1}:[0, a] \rightarrow$ $[0, l]$, we say that $f_{2}$ is a simple expansion of $f_{1}$ if for some $i \quad \exists s:[0, a+$ $\left.n_{i}-1\right] \rightarrow[0, a]$ such that $f_{2}=f_{1} \circ s$, where $s$ is a homeomorphism which has slope 1 everywhere except on some interval $\left[x, x+n_{i}\right], x \in\{0,1, \ldots, a-1\}$, on which it has slope $\frac{1}{n_{i}}$. We think of these expansion maps $s$ as expanding the domain of $f_{1}$ by dividing some unit subinterval of the domain into $n_{i}$ equal pieces and expanding each one to an interval of length one in the domain of $f_{2}$. We extend this to a partial ordering by saying that $f_{1}<f_{2}$ if $f_{2}$ can be obtained from $f_{1}$ by doing finitely many simple expansions. Then $F$ acts on the poset by composition: given $f \in F$ and $g$ in the poset, $f(g)$ is the map $f \circ g$. Since the group acts on the range of a poset element, and expansion takes place in the domain, this action preserves the partial order.

In the case of $F\left(r, \mathbb{Z}\left[\frac{1}{n}\right],\langle n\rangle\right)$, this poset is isomorphic to a subposet of the set of bases of Higman's $V(n, r)$, ordered by expansion (see [9] or [2]). We will therefore call an element of this poset a basis. If an element $f$ has domain $[0, a]$, we will say that $f$ is a basis of size $a$. If $f$ and $g$ are two bases with $f<g$, we will say that $g$ is an expansion of $f$. We will refer to the basis which is just the identity map on $[0, l]$ as the standard basis. Then any expansion of the standard basis of size $a$ takes $\{0,1,2, \ldots, a\}$ to some $P$-regular subdivision of $[0, l]$ and is linear on each subinterval $[i, i+1]$ for $i=0, \ldots, a-1$ (for the definition of $P$-regular subdivision see A.2). So the subposet of all expansions of the standard basis is in one-to-one correspondence with the set of $P$-regular subdivisions of $[0, l]$. Now if $v_{1}$ and $v_{2}$ are any two bases of the same size, then there exists a unique group element $f$ such that $f\left(v_{1}\right)=v_{2}$. Conversely, using the above interpretation of $P$-regular subdivisions, A.2 tells us that given any $f \in F$, there exist $v_{1}$ and $v_{2}$, expansions of the standard basis of the same size, such that $f\left(v_{1}\right)=v_{2}$. Such ordered pairs of bases are the analogues of Higmans' symbols.

We call the subposet of all expansions of the standard basis the standard subposet. Then any group element $g$ translates this whole subposet to an isomorphic one consisting of all expansions of the $g$-translate of the standard basis. Conversely, any basis is contained in some translate of the standard subposet. For if $g:[0, a] \rightarrow[0, l]$ is any basis, any choice of a composition of expansion maps $S:[0, a] \rightarrow[0, l]$ reveals $g$ to be an expansion of $g \circ S^{-1} \in F$, so $g$ lies in the $g \circ S^{-1}$ translate of the standard subposet. We can keep track of the relationships within the standard subposet by associating a forest to each basis. To the standard basis we associate a row of $l$ dots. Then if $f$ is a basis in the standard subposet we can inductively represent the simple expansion of $f$ obtained by expanding the $i$ th $f$-interval into $n_{j}$ by drawing the forest for $f$, and then drawing $n_{j}$ new leaves descending from the $i$ th leaf in the forest for $f$. As an example, we provide the picture for the expansion obtained from the standard basis by dividing the first interval in thirds, and then the second interval of the result in half, with $l=2$ : 


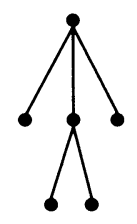

Now given bases $f:[0, a] \rightarrow[0, l]$ and $g:[0, b] \rightarrow[0, l]$, we see by a minor modification of A.3 that $g^{-1} \circ f:[0, a] \rightarrow[0, b]$ takes the $c$ subintervals of some $P$-regular subdivision of $[0, a]$ linearly to the $c$ subintervals of some $P$-regular subdivision $[0, b]$. But we have seen that these subdivisions correspond to expansions $s$ and $t$ of the identity maps on $[0, a]$ to $[0, b]$. Or, in other words, the diagram

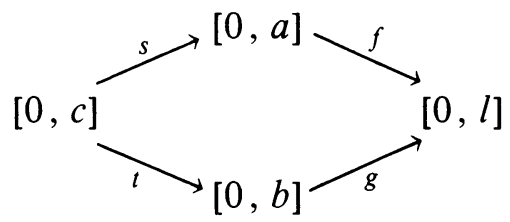

commutes, and $f$ and $g$ have a common expansion in the poset. This means that the poset is directed. Now let $X$ be the simplicial complex associated to the poset; i.e., $X$ has an $n$-simplex for each linearly ordered $(n+1)$-tuple $f_{0}<f_{1}<\cdots<f_{n}$ in the poset. Then $X$ is contractible since the poset is directed, and $F$ acts freely on $X$, so in particular, $X / F$ is a $K(F, 1)$. Now we briefly indicate the changes for the $T$ and $G$ groups.

(a) For the $T$ groups we attempt to imitate what we did for $F$. We take piecewise linear homeomorphisms from $[0, a] /\{0, a\}$ to $[0, l] /\{0, l\}$ which have slopes in $P$, finitely many singularities in $A$, and map $A$ to itself. We can define expansion maps $s$ to be maps from $\left[0, a+n_{i}-1\right] /\left\{0, a+n_{i}-1\right\}$ to $[0, a] /\{0, a\}$ which take 0 to 0 , and have slope 1 everywhere except on some interval $\left[x, x+n_{i}\right]$, on which they have slope $\frac{1}{n_{i}}$. But now the resulting poset is not directed. In particular, if $f$ and $g$ differ by a "rotation," i.e., if $f \circ p=g$, where $p:[0, a] /\{0, a\} \rightarrow[0, a] /\{0, a\}$ takes 0 to $i \in \mathbb{Z}$, and $p$ has slope 1 everywhere, then $f$ and $g$ have no common expansion unless $p$ is the identity. So we modify the poset by taking as bases equivalence classes of the homeomorphisms we chose before, where $f \sim g$ if $f \circ p=g$ for $p$ as above. Then we can say that one equivalence class is a simple expansion of another if they have representatives which are simple expansions of each other in the manner described above. Then everything works, and $T$ acts on the contractible complex $X$. Notice that the action is not free; the vertex stabilizers are finite cyclic groups. Since bases are now equivalence classes, two bases of the same size do not determine a group element. However, given two expansions of the standard basis $v_{1}$ and $v_{2}$ of size $a$, let $g_{1}$ and $g_{2}$ be the representatives in the equivalence classes which are order preserving on $[0, a)$. Then any cyclic permutation $p$ of $a$ elements corresponds to a "rotation" map, and the triple $\left(v_{1}, v_{2}, p\right)$ determines the group element $f=g_{2} \circ p \circ g_{1}^{-1}$. This is the analogue of a symbol of Higman's in this case (see [2] for more explanation).

(b) For the $G$ groups we take as poset elements piecewise linear bijections from $[0, a]$ to $[0, l]$ with finitely many singularities and discontinuities all in $A$ and slopes in $P$. Just as for $T$, the resulting poset is not directed, but this time we need to set $f \sim g$ if $f \circ p=g$, where $p:[0, a) \rightarrow[0, a)$ is allowed 
to arbitrarily permute the subintervals $\{[0,1),[1,2), \ldots,[a-1, a)\}$. The vertex stabilizers for the action of $G$ on $X$ will be finite symmetric groups. Here the symbols which determine group elements consist of two expansions of the standard basis of size $a$ and an arbitrary permutation of $a$ elements.

K. S. Brown [2] (and unpublished) has shown that $\forall n, \exists$ an $n$-connected subcomplex of $X$ on which $F$ (or $T$ or $G$ ) acts with finite quotient complex, which shows that all of these groups are finitely presented and of type $F P_{\infty}$.

We will now describe a homotopy equivalent subcomplex $N$ of $X$ which has an infinite quotient mod the group action, but is useful in computing homology and obtaining presentations for these groups. Again we will talk about the $F$ groups, but everything goes through for $T$ and $G$. In order to define $N$, we will need to study intervals in the poset. In preparation for this, we explain the notation we will use. We will write $[f, g]$ for the closed interval in the poset; i.e., the subposet $\{h \mid f \leq h \leq g\}$. Similarly, we write $(f, g)$ for the open interval. We write $|[f, g]|$ and $|(f, g)|$ for the subcomplexes of $X$ spanned by these intervals. We will refer to all of these things as intervals. When studying such an interval, we are concerned with which expansion maps take you from $f$ to $g$, rather than the particular basis $f$. So we will be looking at the domain of $f$ and how it is divided when expanding to $g$. Given a basis $f:[0, a] \rightarrow[0, l]$, we will refer to the intervals $[i, i+1], i=0,1, \ldots, a-1$, of the domain of $f$ as $f$-intervals. Given $f<f^{\prime}$, every $f^{\prime}$-interval $J^{\prime}$ is mapped by the expansion maps to a subinterval of some $f$-interval $J$, and we can talk about the ratio $l\left(J, J^{\prime}\right)$ of the length of $J$ to the length of the image of $J^{\prime}$ in $J$. Since the $n_{i}$ form a basis for $P$, this is uniquely expressible as a product of the $n_{i}$. Notice that gcd's and lcm's of these ratios exist in the multiplicative monoid generated by the $n_{i}$.

Next we will establish the existence of certain least upper bounds in the poset.

Proposition 1.2 (K. S. Brown). Given a set of simple expansions $\left\{f_{i}\right\}$ of $f$, consider each $f$-interval $J$. Let $m_{J}=1 \mathrm{~cm}$ of the set of $l\left(J, J^{\prime}\right)$, where $J^{\prime}$ ranges over all $f_{i}$-intervals mapping into $J$. Let $h$ be the expansion of $f$ obtained by dividing each $J$ into $m_{J}$ equal pieces. Then $h$ is the least upper bound for the $\left\{f_{i}\right\}$ in the poset.

Proof of 1.2. Clearly $h>f$. Suppose $g$ is another expansion of the $\left\{f_{i}\right\}$. So $g>f$. For each $f$-interval $J$, consider all $g$-intervals $J^{\prime}$ contained in $J$, and let $D_{J}=$ gcd of the set of ratios $l\left(J, J^{\prime}\right)$. Let $g^{\prime}$ be the expansion of $f$ obtained by dividing $J$ into $D_{J}$ even pieces. Then $f<h \leq g^{\prime}$. So we need only show that $g^{\prime} \leq g$.

Lemma 1.3. $g^{\prime} \leq g$.

Proof of 1.3. The proof is by induction on the number of simple expansions of $f$ which are $\leq g$. The conclusion is clear if $g$ is a simple expansion of $f$. Now suppose that $f^{\prime}$ is a simple expansion of $f$ which is less than $g$. Apply the induction hypothesis to $f^{\prime}$ and $g$, obtaining $g^{\prime \prime}$ with $f^{\prime}<g^{\prime \prime} \leq g$. Checking the definitions, we see that $g^{\prime} \leq g^{\prime \prime}$, so $g^{\prime} \leq g$ as well.

Now we are ready to study $X$. It turns out that many of the simplices of $X$ are inessential, in the sense that removing them does not change the homotopy type of $X$. To explain which are essential and which are not, consider an arbitrary interval $[f, g]$. Let $\left\{f_{i}\right\}$ be the simple expansions of $f$ which are in the interval. Let $h$ be the least upper bound of the $f_{i}$. 
Definition 1.4. If $h=g$, then $[f, g]$ (or $(f, g)$ ) is an elementary interval. If $h<g$, the closed and open intervals are nonelementary. Furthermore, a simplex $\left(f_{0}, f_{1}, \ldots, f_{n}\right)$ is elementary if $\left[f_{i}, f_{j}\right]$ is elementary $\forall i<j$. Let $N \subset X$ be the union of all elementary simplices.

Another way to describe an elementary interval is: $\left[f_{i}, f_{j}\right]$ is elementary if in expanding $f_{i}$ to $f_{j}$, each $f_{i}$-interval is divided into $n$ equal pieces, where $n$ is some (possibly empty) product of the $n_{i}$ in which each $n_{i}$ appears at most once. It follows from the definitions that $N$ is an $F$-invariant subcomplex of $X$. It is useful to draw pictures to keep track of what is going on here. Whether or not a simplex is elementary is independent of the particular basis $f_{0}$; it depends only on the expansions involved in getting to the other $f_{i}$. Keeping this in mind, recall that if we represent the basis $f_{0}$ by a row of $a$ dots, where $f_{0}$ has size $a$, then we can represent expansions of $f_{0}$ as forests with $a$ roots. We can then draw a picture of the simplex with forests labelling the vertices. The simplex is then determined by this picture and the basis $f_{0}$. Notice that $F$ will translate the simplex to another simplex with exactly the same picture but a different least vertex. We draw a few examples for the case $F\left(1, \mathbb{Z}\left[\frac{1}{6}\right],\langle 2,3\rangle\right)$ :

Simplices with these pictures are nonelementary:
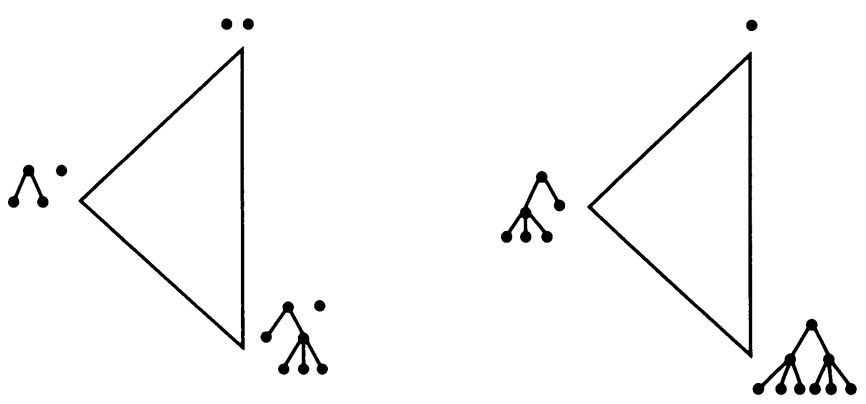

Simplices with these pictures are elementary:
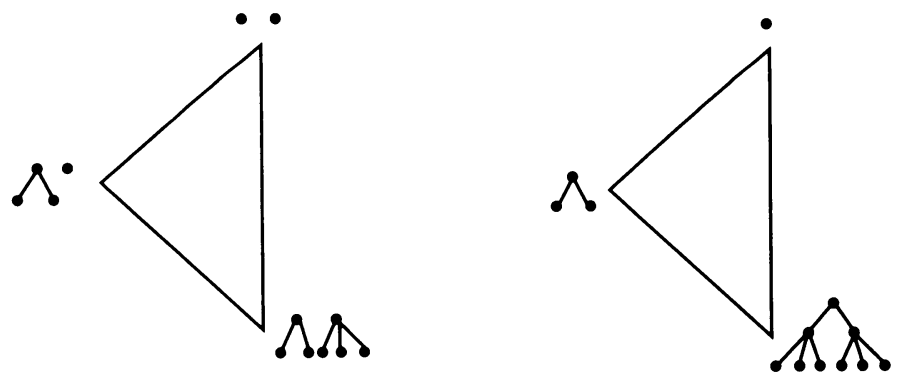

Theorem 1.5. $N$ is contractible.

To prove this, we need to know the homotopy type of $|(f, g)|$ in $X$.

Lemma 1.6. Let $\left\{f_{i}\right\}, 1 \leq i \leq n$, be the simple expansions of $f$ in $(f, g)$. Let $(f, g)_{N}$ be the subposet consisting of all of the lub's of any subset of the $f_{i}$, as long as these lub's are less than $g$. Then the inclusion of $\left|(f, g)_{N}\right|$ into $|(f, g)|$ is a homotopy equivalence. In particular, $|(f, g)|$ is homotopy equivalent to $S^{n-2}$ if the interval is elementary, and is contractible if the interval is nonelementary. 
Proof of 1.6. Make a poset map $p$ from $(f, g)$ to $(f, g)_{N}$ sending any $a$ to the lub of the subset of those $f_{i}$ of which $a$ is an expansion. Since $p$ is a map of posets, $p$ is just the identity on $(f, g)_{N}$, and $p(a) \leq a \forall a, p$ induces a homotopy equivalence between $|(f, g)|$ and $\left|(f, g)_{N}\right|$, with the inclusion map as the homotopy inverse (see [12]). But $\left|(f, g)_{N}\right|$ is isomorphic to the barycentric subdivision of an $(n-1)$-simplex for a nonelementary interval, and is isomorphic to the barycentric subdivision of the boundary of an $(n-1)$ simplex for an elementary interval, which proves the lemma.

We need to make a few remarks for future reference.

(1) Let $[f, g]_{N}=(f, g)_{N} \cup\{f, g\}$. Notice that in the proof above, in the case of an elementary interval we can extend the poset map to $[f, g]$ by fixing $f$ and $g$, which shows that the inclusion $[f, g]_{N} \hookrightarrow[f, g]$ is a homotopy equivalence. Similarly, $\{f, g\} *|(f, g)|_{N} \hookrightarrow\{f, g\} *|(f, g)|$ (where $*$ denotes the join) is a homotopy equivalence.

(2) $\left|(f, g)_{N}\right| \subseteq N$.

(3) If $\sigma=\left(h_{0}, h_{1}, \ldots, h_{r}\right)$ is a simplex of $N$, we claim it is a simplex in $\left|\left[h_{0}, h_{r}\right]_{N}\right|$. Clearly $\left(h_{0}, h_{r}\right)$ is an elementary interval. If $\sigma \notin\left|\left[h_{0}, h_{r}\right]_{N}\right|$, then for some $i, h_{i}$ must not be the lub of a finite collection of simple expansions of $h_{0}$. But then $\left(h_{0}, h_{i}\right)$ is a nonelementary interval, which is not true since $\sigma \in N$.

Proof of 1.5. Let the height of $|(f, g)|$ be $b-a$, where the size of $g$ is $b$ and the size of $f$ is $a$. We can build up $X$ by starting with all of the vertices, then adjoining intervals of height 1 , then all intervals of height 2 , etc. By remarks (2) and (3) above, we can build $N$ by adjoining elementary intervals in order of height; but instead of adjoining the full interval we adjoin $\left|[f, g]_{N}\right|$. Let $X^{n}$ (respectively $N^{n}$ ) be the result of adjoining all intervals of height $\leq n$. Now $N^{0}=X^{0}$. Suppose by induction that $N^{n-1} \hookrightarrow X^{n-1}$ is a homotopy equivalence. We would like to extend this to $N^{n} \hookrightarrow X^{n}$. We do this in two steps. First adjoin all nonelementary intervals of height $n$ to $X^{n-1}$. If $|[f, g]|$ is one such, notice that $|[f, g]| \cap X^{n-1}=\{f, g\} *|(f, g)|$, which is contractible, since it is the suspension of something which is contractible by Lemma 1.6. Since $|[f, g]|$ is also contractible, this adjunction does not change the homotopy type of $X^{n-1}$. Since two height $n$ intervals intersect only in $X^{n-1}$, we can make this argument for each nonelementary interval of height $n$. If we let $X_{1}^{n-1}$ be the result of adjoining all of these intervals to $X^{n-1}$, we have shown that $N^{n-1} \hookrightarrow X_{1}^{n-1}$ is a homotopy equivalence. Now if $(f, g)$ is elementary, we have by remark (1) above the following commutative diagram:

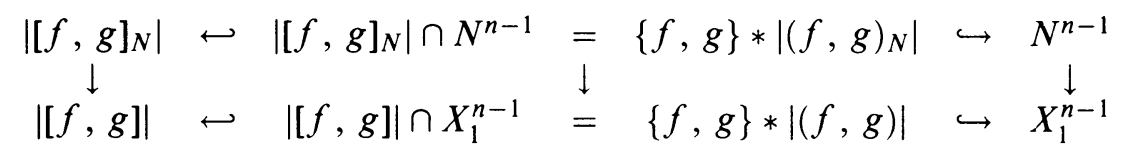

in which the three vertical inclusions are homotopy equivalences. Hence the inclusion

$$
N^{n-1} \cup\left|[f, g]_{N}\right| \hookrightarrow X_{1}^{n-1} \cup|[f, g]|
$$

is a homotopy equivalence $[5,7.5 .7]$. Repeating this for each elementary interval of height $n$, we see that $N^{n} \hookrightarrow X^{n}$ is a homotopy equivalence. So, by induction, $N \hookrightarrow X$ is a homotopy equivalence. But $X$ is contractible, which proves the theorem. 
When we turn to the $T$ and $G$ groups, everything works the same way. In the proof above we were only interested in what goes on inside some interval $(\bar{f}, \bar{g})$, so we can choose $f$ as the representative of $\bar{f}$, and then choose representatives for the other poset elements in the interval which are just $f$ composed with some of the expansion maps. Then the same analysis goes through.

We built up our complex $N$ by adjoining subcomplexes $\left|[f, g]_{N}\right|$. It turns out that each such subcomplex is actually a triangulated $n$-cube, where $n$ is the number of simple expansions of $f$ which are less than $g$. To see this, view $\{0,1\}$ as a poset with $0<1$. Taking the product of $n$ copies of this poset, we obtain a poset $C$ with $n$-tuples of 0 's and 1 's as elements. Then the geometric realization of $C,|C|$, is just a triangulated $n$-cube. We make a poset isomorphism between $C$ and $[f, g]_{N}$, by sending $\left(\varepsilon_{1}, \varepsilon_{2}, \ldots, \varepsilon_{n}\right)$ with $\varepsilon_{i} \in\{0,1\}$ to the least upper bound of $\left\{f_{i} \mid \varepsilon_{i}=1\right\}, i=1,2, \ldots, n$. This reveals $\left|[f, g]_{N}\right|$ to be a triangulated $n$-cube. Any ordering of the $\left\{f_{i}\right\}$ gives such an isomorphism, but we will fix one to use from now on. We let $f_{1}$ be the simple expansion occurring at the leftmost $f$-interval, $f_{2}$ be the one occurring at the next interval to the right, etc. If $f_{i}$ and $f_{j}$ both occur in the same $f$-interval, then $f_{i}<f_{j}$ if $f_{i}$ is an expansion into $n_{i}$ pieces, and $f_{j}$ is an expansion into $n_{j}$ pieces, with $n_{i}<n_{j}$. We will call the resulting isomorphism the characteristic isomorphism. Now if $P$ has rank 1 as an abelian group, any two of these cubes intersect in a common face, so that the cubes give a CWcomplex structure to $N$. Moreover, the $F$ groups act freely on $N$, so in the case of an $F$ group with rank one slope group, the cubical chain complex for $N$ will give a resolution for $F$ with one copy of $\mathbb{Z} F$ in dimension $n$ for each $F$-orbit of $n$-cubes.

For larger rank $P$, however, faces of the cubes may be attached to the interiors of the same or higher dimensional cubes. As an example, the following is a diagram for a square in the complex for $F\left(1, \mathbb{Z}\left[\frac{1}{6}\right],\langle 2,3\rangle\right)$ :

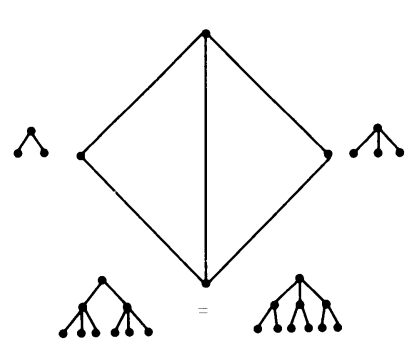

Notice that the two sides attached to the vertex with one dot are two of these cubes, but the other two sides are diagonals of a two-dimensional and a threedimensional cube. The problem with this is that we cannot just write down a chain complex with the cubes as generators; we would have to use simplices instead. Then we have many more cells and a complicated boundary operator, making homology computations difficult.

For the purpose of obtaining a presentation for $F$, however, we can use the cell structure of $N$ given by the cubes. We first choose a maximal tree in the quotient complex consisting only of edges corresponding to simple expansions, and then we have one generator for each $F$-orbit of intervals $[f, g]$, where $g$ is a simple expansion of $f$ (these are the one-dimensional cubes of $N$ ). Then 
we choose a lift of the tree to $N$, which gives us specific homeomorphisms which these generators correspond to. We do not need generators for the other edges because they are all diagonals of cubes, so the homeomorphisms which they correspond to can be expressed in terms of our generating set. We then get a relation for each $F$-orbit of intervals $[f, g]$, where $f$ has two simple expansions which are $\leq g$ (these are the two-dimensional cubes of $N$ ). Since the boundary of some two-cubes contains diagonal edges of higher dimensional cubes, we need to choose a path beginning and ending at the endpoints of the diagonal which goes over only one-dimensional cubes of $N$ in order to express the relation in terms of our generators. If we do this systematically, we obtain regular looking presentations for any of our $F$ groups. For $T$ and $G$ groups, we do a similar thing, but we also need to add to our presentation stabilizers of the bases on the tree and certain conjugation relations among them (see [4]).

Example 1. For $F\left(1, \mathbb{Z}\left[\frac{1}{2}\right],\langle 2\rangle\right), N$ is actually a cubical complex. We lift a maximal tree in $N / F$ to $N$ by lifting the vertex of size one in $N / F$ to the standard basis, and then making a tree by taking successive expansions at the leftmost interval. From this we obtain the following presentation:

$$
\left\langle x_{0}, x_{1}, x_{2}, \ldots \mid x_{j} x_{i}=x_{i} x_{j+1} \forall i<j\right\rangle .
$$

This is the well-known presentation for this group [1].

Remark. In this example, as for any $F$ group with $P$ generated by one integer, Brown constructs essentially the same resolution as the one given by the chain complex of $N$ in a different way, using the known presentation and viewing it as a rewriting system for words in the generators (see [3]). This method does not seem to generalize to more than one integer.

Example 2. For $F\left(1, \mathbb{Z}\left[\frac{1}{6}\right],\langle 2,3\rangle\right)$, using the same tree as above, we obtain

$$
\begin{aligned}
&\left\langle x_{0}, x_{1}, \ldots ; y_{0}, y_{1}, \ldots ; y_{0}^{+}, y_{1}^{+}, \ldots\right| x_{j} x_{i}=x_{i} x_{j+1}, y_{j} x_{i}=x_{i} y_{j+1}, \\
& y_{j}^{+} x_{i}=x_{i} y_{j+1}^{+}, x_{j} y_{i}^{+}=y_{i}^{+} x_{j+2}, \\
& y_{j} y_{i}^{+}=y_{i}^{+} y_{j+2}, y_{j}^{+} y_{i}^{+}=y_{i}^{+} y_{j+2}^{+} \forall i<j ; \\
&\left.y_{i+1} y_{i}^{+}=y_{i} x_{i+1} x_{i}, x_{i} y_{i+1}^{+} y_{i}^{+}=y_{i}^{+} x_{i+2} x_{i+1} x_{i} \forall i\right\rangle .
\end{aligned}
$$

In both examples,

$$
a_{i+1}(t)= \begin{cases}\frac{1}{2} a_{i}(2 t) & \text { if } t \in\left[0, \frac{1}{2}\right] \\ t & \text { if } t \in\left[\frac{1}{2}, 1\right]\end{cases}
$$

where $t \in[0,1]$ and $a=x, y$, or $y^{+}$, and $x_{0}, y_{0}$, and $y_{0}^{+}$are the homeomorphisms graphed below:
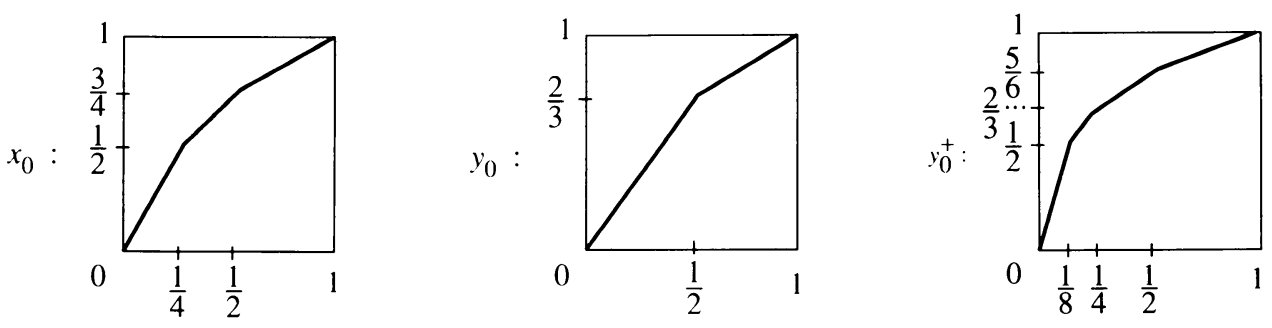


\section{COLlAPSING TO COMPLEXES OF FINITE TYPE}

In this section we restrict ourselves to the groups $F\left(l, \mathbb{Z}\left[\frac{1}{n_{1} \cdots n_{k}}\right],\left\langle n_{1}, \ldots, n_{k}\right\rangle\right)$, and show how to collapse the complex $N / F$ to a homotopy equivalent complex with finitely many cells in each dimension. We follow the method described in $[3,1]$. Recall that $d=\operatorname{gcd}\left(n_{1}-1, \ldots, n_{k}-1\right)$, and assume that $n_{1}<n_{2}<\cdots<n_{k}$. We need to set up some notation before we begin.

We will view $N$ as a "cell complex" with the cubes $\left|[f, g]_{N}\right|$ as the cells. Remember that with this cell structure, the attaching maps do not always go into lower dimensional skeleta, so this is not a CW-complex structure. This means that we cannot build $N$ by adjoining cells in order of dimension. There is a natural concept, called degree, which replaces the notion of dimension. For any cell $\left|[f, g]_{N}\right|$, each $f$-interval is expanded into $p$ pieces, where $p$ is a product of the $n_{i}$ in which each $n_{i}$ appears at most once. We say that $\left|[f, g]_{N}\right|$ has degree $\left(a_{1}, \ldots, a_{k}\right)$, where $a_{j}$ is the number of $f$-intervals which are divided into $p$ pieces; here $p$ is a product of $k-j+1$ of the $n_{i}$. Then the dimension of a cell of degree $\left(a_{1}, \ldots, a_{k}\right)$ is $k a_{1}+(k-1) a_{2}+\cdots+3 a_{k-2}+2 a_{k-1}+a_{k}$. The degrees may be ordered lexicographically. Then we note that each geometric face of $\left|[f, g]_{N}\right|$ is contained in a cell of smaller degree, even though it may have larger dimension. Thus we can build $N$ by adjoining cells in order of degree. Notice that in the case $k=1$, the degree of a cell is just a one-tuple which is the dimension of the cell, so this order of adjunction is just the standard order of adjoining the cells in a $\mathrm{CW}$-complex.

The action of $F$ is cellular and preserves both dimension and degree. If we look at the action of $F$ on one cell $\sigma$ of $N$, we see that the only set of points of $\sigma$ which are identified are zero cells, so the quotient complex consists of cubes with perhaps some 0 -faces identified. We will give each such cube in the quotient complex a symbol. First notice that there is one zero cell for each natural number $s \equiv l(\bmod d)$ (corresponding to all bases of size $s)$. We call the zero cell corresponding to the number $s, v^{s}$ (we will also write this as a string of $s v$ 's, and will call this a symbol of length $s$ ). Now for each $n$-cell $\sigma$ in the quotient we may choose a lift $[f, g]_{N}$ in $N . g$ is obtained by expanding certain $f$-intervals into $p$ pieces, where $p$ is a product of the $n_{i}$ in which each $n_{i}$ appears at most once. We encode this information in a symbol of length $s$, where $s$ is the number of $f$-intervals. Our symbol has one letter for each $f$ interval, with the leftmost letter corresponding to the leftmost $f$-interval, etc. We put $v$ if there is no expansion at an interval, and $x_{J}$ if the interval is divided into $n_{i_{1}} n_{i_{2}} \cdots n_{i_{r}}$ equal pieces, where $J=\left\{i_{1}, i_{2}, \ldots, i_{r}\right\}$. In the case $k=1$, we may omit the subscripts on $x$. This symbol is independent of the choice of lift of $\sigma$. For example, $v^{2} x_{1} x_{1,2} v$ is a three-cell of $N / F$ such that any lift of it to $N$, written $[f, g]_{N}$, has $f$ a basis of size 5 . When expanding from $f$ to $g$, the third $f$-interval, $[2,3]$, is divided into $n_{1}$ equal pieces; and the fourth $f$-interval, $[3,4]$, is divided into $n_{1} n_{2}$ equal pieces.

Notice that these symbols are just another way of writing the forest symbols we used in the last section. If we are given a cube $[f, g]_{N} \in N$, we associated a forest to $g$ which described how it was related to $f$ in the poset. The forest had $s$ roots, where $f$ was a basis of size $s$, and each letter used above corresponds to a tree at a single root. Notice that we only have a finite number of possible such trees because $(f, g)$ is elementary. 
We now define face operators on the cells of $N / F$. An $n$-cell $\sigma$ will have $2 n$ faces, $A_{i}(\sigma)$ and $B_{i}(\sigma)$ for $i \in\{1, \ldots, n\}$. Suppose $\sigma=S x_{J} R$, where $S$ is an $s$-cell and $J=\left\{i_{1}, i_{2}, \ldots, i_{r}\right\}$, with $r \geq 1$ and $i_{1}<\cdots<i_{r}$. We may think of $v$ as $x_{\varnothing}$ for this definition. Choose $j$ with $1 \leq j \leq r$, and let $J^{\prime}=J \backslash\left\{i_{j}\right\}$. Then we define

$$
A_{s+j}(\sigma)=S x_{J^{\prime}} R, \quad B_{s+j}(\sigma)=S \underbrace{x_{J^{\prime}} \cdots x_{J^{\prime}}}_{n_{i j}} R .
$$

Now each $n$-cell is a cube, with $2 n$ geometric faces which are $(n-1)$-cubes, though not necessarily $n$-cells. Choosing any lift of $\sigma$ to $N$, say $\tau$, we have a characteristic isomorphism from $I^{n}$ to $\tau$. Each face $\sigma_{1}$ of $\sigma$ is the image of a face of $I^{n}$ under the composition of the characteristic isomorphism and the quotient map. We call $\sigma_{1}$ the geometric $A_{i}$ or $B_{i}$ face of $\sigma$ if it is the image of the $A_{i}$ or $B_{i}$ face of $I^{n}$. The $A_{i}$-faces of a cell defined above are precisely the geometric $A_{i}$-faces of the cell, whereas the $B_{i}$-faces defined above are the geometric $B_{i}$-faces of the cell only if $r=1$. Otherwise, the geometric $B_{i}$-faces of the cell are diagonals of the $B_{i}$-faces defined above.

We are now going to classify the cells as essential, redundant, or collapsible. We will then give a bijection $c$ from the set of redundant cells of degree $\left(a_{1}, \ldots, a_{k}\right)$ to the set of collapsible cells of degree $\left(a_{1}, a_{2}, \ldots, a_{k-1}, a_{k}+1\right)$ such that $\sigma$ is a geometric face of $c(\sigma)$. We then build up our space by a series of adjunctions, first adjoining all essential cells of a given degree $a$ and then attaching each redundant cell $\sigma$ of degree $a$ along with $c(\sigma)$. We then move on to the next greatest degree and repeat the process. Our setup will ensure that there is some order in which to adjoin the redundant cells of a given degree such that when we adjoin $\sigma$, all other faces of the collapsible cell $c(\sigma)$ will have been already adjoined. Then this adjunction will just be an elementary expansion, and will not change the homotopy type. This argument yields a quotient complex $Y$ of $N / F$ with one cell for each essential cell of $N / F$, where the quotient map is a homotopy equivalence. For more detail, see [3] or [1].

Definition 2.1. A collapsible pattern is an $x_{1}$ not preceded by $v$. A redundant pattern is $n_{1} v$ 's in a row. A cell is essential if it contains no collapsible or redundant pattern. An inessential cell is collapsible if the first (always from the left) such pattern is collapsible, and redundant if the first such pattern is redundant.

Definition 2.2. Let $\sigma$ be a redundant cell of degree $a$. Then $\sigma=R v^{n_{1}} S$, where $R$ is a symbol with no redundant or collapsible patterns which does not end in $v$. Let $c(\sigma)=R x_{1} S$.

One can now verify the following:

Claim 2.3. $c$ gives a bijection between the set of redundant cells of degree $a=\left(a_{1}, \ldots, a_{k}\right)$ and the set of collapsible cells of degree $\left(a_{1}, \ldots, a_{k}+1\right)$, such that $\sigma$ is a geometric face of $c(\sigma)$, and all other faces of $c(\sigma)$ are $\neq \sigma$. In addition, suppose $\sigma$ has degree $a=\left(a_{1}, \ldots, a_{k}\right)$, and let $\tau \neq \sigma$ be another face of $c(\sigma)$. Then $\tau$ has degree $a^{\prime}=\left(a_{1}^{\prime}, \ldots, a_{k}^{\prime}\right)$ with $a^{\prime} \leq a$. So if $\tau$ is essential, $\tau$ was adjoined before $\sigma$. If $\tau$ is collapsible, then $c^{-1}(\tau)$ has degree $\left(a_{1}^{\prime}, \ldots, a_{k}^{\prime}-1\right)<a$, so that $\tau$ was adjoined before $\sigma$. 
We call $\tau^{\prime}$ an immediate predecessor of $\tau$ if $\tau^{\prime}$ is another redundant face of $c(\tau)$ with the same degree as $\tau$. We now need to show that there is some order in which to attach the redundant cells of a given degree, along with their collapsible partners, which ensures that all the immediate predecessors of $\tau$ have already been adjoined when we adjoin $\tau$. We will first show that there are no infinite chains of immediate predecessors, by which we mean a sequence $\tau_{1}, \tau_{2}, \tau_{3}, \ldots$ of redundant cells such that $\tau_{i+1}$ is an immediate predecessor of $\tau_{i} \forall i$. If this is true, then any set of cells of degree $a$ contains minimal elements, where a minimal element is one which has no immediate predecessors. We can therefore factor the adjunction of the redundant cells of degree $a$ into stages. We first adjoin all minimal redundant cells, along with their collapsible partners. We then attach the minimal cells amongst the remaining redundant cells, and so on. This ensures that if we build our complex in the manner described above, all steps except the adjunctions of essential cells are elementary expansions.

To show that there are no infinite chains, we first define $\chi(\tau)$ to be the symbol for $\tau$ with all of the $v$ 's deleted. We now put a partial order on the $\chi$ 's by setting $x_{1}<x_{J}$, whenever $J \neq\{1\}$, and ordering the $\chi$ 's lexicographically, reading from the right. Since the length of $\chi(\tau)$ for $\tau$ of degree $a$ is at most $a$, this partial order has no infinite descending chains of $\chi$ 's for cells of degree $a$. Then we have:

Claim 2.4. Suppose $\tau^{\prime}$ is an immediate predecessor of $\tau$. Then either (1) $\chi\left(\tau^{\prime}\right)<\chi(\tau)$, or $(2) \chi\left(\tau^{\prime}\right)=\chi(\tau)$, in which case $\tau^{\prime}=R v S$ and $\tau=R v^{n_{1}} S$.

It follows that there are no infinite chains of immediate predecessors. So we see that we can carry out this collapse of our complex onto a quotient complex $Y$ with one cell for each essential cell. Checking the definition of essential, we see that the number of $v$ 's in an essential symbol of a given dimension is bounded, so there are only finitely many essential symbols in each dimension. So $Y$ is a $K(F, 1)$ with finitely many cells in each dimension, establishing:

Theorem 2.5. $F\left(l, \mathbb{Z}\left[\frac{1}{n_{1} \cdots n_{k}}\right],\left\langle n_{1}, \ldots, n_{k}\right\rangle\right)$ is finitely presented and of type $F P_{\infty}$.

This has been shown in a more abstract way using the complex $X$ by K. S. Brown (unpublished).

Now recall that if the slope group is generated by only one integer, the attaching maps for an $n$-cube of $N$ do go into the $(n-1)$-skeleton, and the degree of an $n$-cell is just $(n)$. So we are just thinking of $N$ as a CW-complex with the cubes as cells. $Y$ is therefore a CW-complex with one cell for each essential cell of $N / F$. Since $N$ is a regular CW-complex, it is easy to compute the boundary operator in the chain complex of $N / F$. Each cell which is in the image of the attaching map must appear with coefficient \pm 1 . In this case, the face operators in $N$ always give the geometric faces, and one checks that the face operators satisfy the usual identities for cubical face operators. Therefore, if we set $\partial(\sigma)=\sum(-1)^{i}\left(A_{i}(\sigma)-B_{i}(\sigma)\right)$, then $\partial^{2}=0$. It follows that this is the boundary operator in the chain complex of $N / F$ (see [6, Chapter 3]). The chain complex for $Y$ is obtained by taking the quotient complex of this by the subcomplex generated by the collapsible cells.

To compute the boundary in the chain complex of $Y$, we choose as a lift of a given cell of $Y$ the corresponding essential cell $\sigma$ of $N / F$, and compute its boundary. Any collapsible cells which appear in $\partial(\sigma)$ will be zero in the 
quotient complex, so we can ignore them. For each redundant cell $\tau$, we find $c(\tau)$, and substitute $\tau-\partial(c(\tau))$ for $\tau$ in the formula for $\partial(\sigma)$. Again, any new collapsible cells may be ignored, and we keep replacing redundant cells in this manner. The process will end (because a cell has only finitely many immediate predecessors, and there are no infinite chains of immediate predecessors) with only essential cells in the expression for $\partial(\sigma)$; and the corresponding cells in $Y$, with the same coefficients, will be the boundary of the cell we started with. One can then check that if $\sigma$ is an essential cell of $N / F, A_{i}(\sigma)-B_{i}(\sigma)=0$ in the quotient chain complex, by induction on the number of non- $v$ 's to the left of the $x_{1}$ at which the $i$ th faces occur. But then the boundary maps in the quotient complex are zero, so the homology of $F$ is just computed by counting the essential cells, yielding

Theorem 2.6. If $F_{i}=F\left(i, \mathbb{Z}\left[\frac{1}{n}\right],\langle n\rangle\right)$, then $H_{k}\left(F_{i}\right)=\mathbb{Z}^{n(n-1)^{k-1}}$ for $k \geq 1$.

We record here the finite presentation provided by this collapse which corresponds to one of the infinite presentations we gave in $\S 1$.

Example 1. For $F\left(1, \mathbb{Z}\left[\frac{1}{2}\right],\langle 2\rangle\right)$ we have

$$
\left.\left\langle x_{0}, x_{1}\right| x_{2} x_{0}=x_{0} x_{3}, x_{3} x_{1}=x_{1} x_{4}\right),
$$

where $x_{i-2}^{-1} x_{i-1} x_{i-2}=x_{i} \quad \forall i \geq 2$.

We will not give the finite presentation corresponding to our infinite presentation for $F\left(1, \mathbb{Z}\left[\frac{1}{6}\right],\langle 2,3\rangle\right)$, which has six generators and 22 relations, because in $\S 3$ we will do a more complete collapse and obtain a smaller one consisting of four generators and ten relations. This will be shown to be the most economical one possible.

We remark that the homology result above, as well as the corresponding finiteness results for a slope group generated by one integer have been obtained by C. Squier (unpublished). He constructs essentially the same chain complex by a different method, making use of the known infinite presentation for the one integer case. The result in the case of slope group generated by 2 is due to K. S. Brown and R. Geoghegan [1], also using the known presentation and yet another method to construct essentially the same resolution. The homology results can also be deduced from work of P. Greenberg [8]. The methods here are completely different, coming from the theory of foliations. They lead to homology results in this and other cases but do not give presentations or information about finiteness properties.

\section{More COllaPSing}

In the last section we saw how a collapse of the complex $N$ led easily to a computation of the homology of $F$ in the case where the slope group of $F$ has rank one. For a slope group of higher rank, the boundary maps are not zero, so to compute the homology of $F$ from $Y$ would involve some work. In this section we will show that we can do much more collapsing, provided that we replace $N$ by a homotopy equivalent complex $K$. The latter will be a CWcomplex with one cell for each cube of $N$. Recall that $N$ is not a CW-complex with the cubes as cells because some of its cubes have faces which lie in higher dimensional cubes. 
We begin by setting up the collapsing scheme we would like to use. We classify the cubes of $N / F$ as collapsible, redundant, or essential. First recall from $\S 2$ that we have symbols for the cells which are words in the letters $v$ and $x_{J}$, where $J$ is some subset of $\{1,2, \ldots, k\}$, and $k$ is the rank of the slope group. In the following two definitions, we may think of $v$ as $x_{J}$, where $J=\varnothing$. Elsewhere, we will always mean $J$ to be nonempty.

Definition 3.1. A collapsible pattern is an $x_{J}$ not preceded by $v$, where $1 \in J$. A redundant pattern is $n_{1}-1=r_{1} \quad v$ 's followed by $x_{J}$ where $1 \notin J$. A cell is essential if it contains no collapsible or redundant patterns. An inessential cell is collapsible if the first such pattern is collapsible, and redundant if the first such pattern is redundant.

Definition 3.2. Let $\sigma$ be a redundant $m$-cube. Then $\sigma=R v^{r_{1}} x_{J} S$, where $R$ is an essential cube and $1 \notin J$. Then let $c(\sigma)=R x_{J \cup\{1\}} S$.

Then one easily verifies:

Claim 3.3. $c$ is a bijection between the set of redundant $m$-cells and the set of collapsible $(m+1)$-cells. If $\sigma$ is redundant, $\sigma$ is contained in a face of $c(\sigma)$, where here we mean face as defined in $\S 2$.

Keeping in mind that the cubes of $K$ are going to give a CW-complex structure to $K$, we are going to build up $K$ in order of dimension, instead of degree. So we will first adjoin all essential $m$-cells, then the redundant $m$-cells together with their collapsible partners, and then move on to dimension $m+1$.

In order to carry out the collapse as in $\S 2$, we need $\sigma$ to be a geometric face of $c(\sigma)$. We also will need to know that there is some order in which to attach the redundant $m$-cells such that all other cells on the boundary of $c(\sigma)$ (which are all $m$-cells) are adjoined before $\sigma$. If the cells are essential or collapsible, this is now automatic. So if we say that $\sigma^{\prime}$ is an immediate predecessor of $\sigma$ if it is another redundant face of $c(\sigma)$, then we need to show that there are no infinite chains of immediate predecessors. Then it will follow exactly as in $\S 2$ that we will be able to carry out our collapse.

We will now show

Theorem 3.4. There is $K(F, 1)$ which is a $C W$-complex with one cell for each cube of $N / F$. Hence we can classify the cells of this $C W$-complex as redundant, collapsible, and essential, using the one-to-one correspondence between the cells and the cubes of $N / F$. Then the complex has the property that if $\sigma$ is a redundant cell, then $\sigma$ is a geometric face of $c(\sigma)$. In addition, there are no infinite chains of immediate predecessors.

We will prove the theorem by constructing a contractible, regular CW-complex $K$ which has one cell for each cube of $N$, such that $F$ acts freely on $K$ and $K / F$ has the properties in the statement of the theorem. We are going to construct $K$ by adjoining an $m$-cube $\sigma^{\prime}$ for each $m$-cube $\sigma$ of $N$. We will adjoin the $\sigma^{\prime}$ in order of the degree of $\sigma$.

In preparation for describing our attaching maps, we define face operators for the cube $\left|[f, g]_{N}\right|$ as in $\S 2$. These are just the lifts of the face operators of $N / F$ to $N$. Let $d_{1}, d_{2}, \ldots, d_{n}$ be the simple expansions of $f$ which are less than $g$, in the standard order. Set

$$
\hat{d}_{i}=\text { lub of }\left\{d_{1}, d_{2}, \ldots, \hat{d}_{i}, \ldots, d_{n}\right\} .
$$


We then set $A_{i}\left(\left|[f, g]_{N}\right|\right)=\left|\left[f, \hat{d}_{i}\right]_{N}\right|$ and $B_{i}\left(\left|[f, g]_{N}\right|\right)=\left|\left[d_{i}, g\right]_{N}\right|$. One checks that if $\tau$ is a face of $\sigma$, then $\tau$ has smaller degree than $\sigma$. As before, the geometric faces of the cube $\left|[f, g]_{N}\right|$ have names $A_{i}$ and $B_{i}$ coming from the characteristic isomorphism from $I^{n}$ to $\left|[f, g]_{N}\right|$. Notice that the $A_{i}$ faces always coincide with the geometric faces of the cube, whereas the $B_{i}$ faces only coincide with the geometric faces if $d_{i}$ corresponds to an $f$-interval which is divided into exactly $n_{j}$ pieces, for some $j$, in expanding $f$ to $g$. An interval divided into $p$ pieces, where $p$ is the product of more than one of the $n_{j}$, gives $B_{i}$ and $B_{i+1}$ faces which are cubes containing the geometric faces as diagonals.

In order to construct $K$, we also need to define some maps between standard cubes. First we define $d_{2}^{\prime}: I \rightarrow I \times I$ by

$$
d_{2}^{\prime}(t)= \begin{cases}(0, t), & t \in\left[0, \frac{1}{2}\right] \\ (2 t-1,1), & t \in\left[\frac{1}{2}, 1\right] .\end{cases}
$$

We will use this to move the diagonal of a square out to a path in the boundary. Now we extend this to one-dimensional diagonals of higher dimensional cubes. Define $d_{n}^{\prime}: I \rightarrow I^{n}$ for $n>2$ inductively by

$$
d_{n}^{\prime}(t)=\left(d_{2}^{\prime} \times \operatorname{id}_{I^{n-2}}\right) \circ d_{n-1}^{\prime} .
$$

In order for things to fit together properly, we have to change scale here. We define

$$
d_{n}=d_{n}^{\prime} \circ c_{n},
$$

where $c_{n}: I \rightarrow I$ is just the piecewise linear homeomorphism of $I$ which takes the subdivision $\left(0, \frac{1}{n}, \frac{2}{n}, \ldots, \frac{n-1}{n}, 1\right)$ linearly to $\left(0, \frac{1}{2}, \frac{3}{4}, \ldots, \frac{2^{n-1}-1}{2^{n-1}}, 1\right)$. Now we extend to maps for higher dimensional diagonals by defining $d_{j, n}: I^{j} \rightarrow$ $\left(I^{j}\right)^{n}$ by

$$
d_{j, n}=T_{j, n} \circ\left(d_{n}\right)^{j},
$$

where $T_{j, n}: I^{n j} \rightarrow I^{n j}$ just shuffles the coordinates by sending

$$
\left(a_{11}, \ldots, a_{1 n} ; \ldots ; a_{j 1}, \ldots, a_{j n}\right) \rightarrow\left(a_{11}, \ldots, a_{j 1} ; \ldots ; a_{1 n}, \ldots, a_{j n}\right) .
$$

We are now ready to construct $K$. For each $m$-cube $\sigma$ of $N$ we adjoin a copy of $I^{m}, \sigma^{\prime}$, to $K$. We adjoin in order of the degree of $\sigma^{\prime}$, and after each adjunction of a cell $\sigma^{\prime}$ we will have a characteristic map from $I^{m}$ to $\sigma^{\prime} \in K$.

Now consider an $m$-cube $\sigma$ of $N$, and suppose that we have already adjoined cells to $K$ for the cubes of lower degree in $N$. In order to adjoin a copy of $I^{m}$, which will be called $\sigma^{\prime}$, we need an attaching map from the boundary of $I^{m}$ to the part of $K$ which has already been constructed. We describe this map one face at a time. If, for some $i$, the geometric $A_{i}$ (or $B_{i}$ ) face of $\sigma$ coincides with $A_{i}(\sigma)$ (or $\left.B_{i}(\sigma)\right)$ in $N$, we will just use the characteristic map for $\left(A_{i}(\sigma)\right)^{\prime}$ (or $\left.\left(B_{i}(\sigma)\right)^{\prime}\right)$ as our attaching map. We have this map already since $A_{i}(\sigma)$ (or $\left.B_{i}(\sigma)\right)$ has lower degree than $\sigma$.

This takes care of all of the faces except some $B$ faces. Suppose $\sigma$ is a cube of $N$ with image $S x_{J} R$ in $N / F$, where $R$ is an $r$-cube, $S$ is an $s$-cube, $J$ has $i$ elements with $i \geq 2$, and $r+i+s=m$. Let $n$ be $n_{t}$, where $t$ is the $j$ th smallest integer in $J$. For $1 \leq j \leq i$, the geometric $B_{s+j}$ face of $\sigma$ is a diagonal of $B_{s+j}(\sigma)$, so we need to explain how to attach the $B_{s+j}$ face of $I^{m}$. It is just an $(m-1)$-cube, which we write $I^{s} \times I^{i-1} \times I^{r}$. We attach it via

$$
I^{s} \times I^{i-1} \times I^{r} \rightarrow I^{s} \times\left(I^{i-1}\right)^{n} \times I^{r} \rightarrow\left(B_{s+j}(\sigma)\right)^{\prime},
$$


where the first map is $\operatorname{id}_{I^{s}} \times d_{i-1, n} \times \operatorname{id}_{I^{r}}$, and the second map is the characteristic map for $\left(B_{s+j}(\sigma)\right)^{\prime}$. As before, $B_{s+j}(\sigma)$ has lower degree than $\sigma$, so if we attach in order of degree this attaching map makes sense. Now one checks that the maps agree on the intersections of faces. This is almost immediate for two faces corresponding to two faces of $\sigma$ arising from different letters in the symbol for the image of $\sigma$ in $N / F$. Agreement on the intersection of an $A$ and a $B$ face arising from the same letter is also easy to see. For the case of two $B$ faces arising from the same letter, agreement on the intersection follows from the fact that

$$
\left(d_{i, p}\right)^{m} \circ d_{i, m}=d_{i, p m}=\left(d_{i, m}\right)^{p} \circ d_{i, p} .
$$

These attaching maps give a one-to-one map from the boundary of an $m$-cell to a union of $(m-1)$-cells of smaller degree. One can check inductively that the characteristic maps are isomorphisms, so that $K$ is a regular CW-complex with the interiors of the cubes partitioning the points of $K$ into open cells.

Now we claim that $K$ is contractible. In fact, this does not depend on any choices we made in defining the $d_{j, n}$, but only on the fact that $K$ has one cell $\sigma^{\prime}$ for each cube $\sigma$ of $N$, with boundary contained within the union of the $\left(B_{i}(\sigma)\right)^{\prime}$ and the $\left(A_{i}(\sigma)\right)^{\prime}$. For we have:

Proposition 3.5. $K$ is homotopy equivalent to $N$.

Proof of 3.5. We prove this by contractible carriers [11, Chapter 2, Theorem 9.2]. Let $a$ be a simplex in $N$, say $a=\left(f_{0}, \ldots, f_{n}\right)$ with $f_{0}<f_{1}<\cdots<f_{n}$, so $a \subseteq\left|\left[f_{0}, f_{n}\right]_{N}\right|=\sigma(a)$. Let $d_{1}, \ldots, d_{r}$ be the simple expansions of $f_{0}$ which are less than $f_{n}$. Let

$$
\mathscr{C}^{\prime}(a)=(\sigma(a))^{\prime} \cup \bigcup_{i=1}^{r}\left[B_{i}(\sigma(a))\right]^{\prime} \subset K .
$$

$\mathscr{C}^{\prime}(a)$ may be built by gluing the cubes in the union to the main cube $(\sigma(a))^{\prime}$ via the attaching maps. One checks that these cubes can be adjoined one by one along contractible intersections, so that $\mathscr{C}^{\prime}(a)$ is contractible. On the other hand, given a cell $b^{\prime}$ of $K$ corresponding to a cube $b$ of $N$, let

$$
\mathscr{C}\left(b^{\prime}\right)=b \cup\left(\bigcup_{i=1}^{t} B_{i}(b)\right) \subset N .
$$

Now there exists $\left|\left[g_{0}, g_{s}\right]\right| \hookrightarrow X$ such that $\mathscr{C}\left(b^{\prime}\right) \hookrightarrow \mid\left[g_{0}, g_{s}\right]$. But we had a retraction of $\left|\left[g_{0}, g_{s}\right]\right|$ onto $\left|\left[g_{0}, g_{s}\right]_{N}\right|=b$ inside $X$. Under this retraction $B_{i}(b)$ stayed inside itself, so the retraction restricted to $\mathscr{C}\left(b^{\prime}\right)$ takes place within $N$. $\mathscr{C}\left(b^{\prime}\right)$ retracts to $b$, which is contractible. We now have two systems of contractible subcomplexes in $K$ and $N$ :

$$
\left\{\mathscr{C}\left(b^{\prime}\right)\right\}_{b^{\prime} \in K} \text { and }\left\{\mathscr{C}^{\prime}(a)\right\}_{a \in N} .
$$

Now by construction if $a_{1}$ is a face of $a, \mathscr{C}^{\prime}\left(a_{1}\right) \subseteq \mathscr{C}^{\prime}(a)$; and if $b_{1}^{\prime}$ is a face of $b^{\prime}, \mathscr{C}\left(b_{1}^{\prime}\right) \subseteq \mathscr{C}\left(b^{\prime}\right)$. So there exist maps $\alpha: N \rightarrow K$ and $\beta: K \rightarrow N$ carried by these systems of contractible complexes. Notice that if $b^{\prime} \in K, \alpha \circ \beta\left(b^{\prime}\right)$ is contained in $b^{\prime} \cup\left(\bigcup\left[B_{i}(b)\right]\right)^{\prime}=\mathscr{C}^{\prime}(b)$, which also contains $\operatorname{id}_{K}\left(b^{\prime}\right)=b^{\prime}$. This shows that $\alpha \circ \beta \simeq \operatorname{id}_{K}$. Similarly, $\beta \circ \alpha \simeq \operatorname{id}_{N}$, and so $N \simeq K$.

Notice that the vertices of $K$ are in one-to-one correspondence with the vertices of $N$, so this gives an action of $F$ on the vertices of $K$. But the group 
action on all of $N$ takes cubes to cubes, and one checks that this induces a free cellular action on the whole complex $K$ extending the action on the vertices. This means that the quotient complex $K / F$ is a $K(F, 1)$. If $h \in F$, then $(h(\sigma))^{\prime}=h\left(\sigma^{\prime}\right)$, so $K / F$ has one cell for each cube in $N / F$.

We will now drop the primes and use the symbols for the cubes of $N / F$ for the corresponding cells of $K / F$. To check that $K / F$ has $\sigma$ as a face of $c(\sigma)$ for any redundant cell $\sigma$, we need to figure out which cells are on the geometric faces of our cubes. This can be done by examining the attaching maps. We will later on give the explicit formula for the boundary maps in the cellular chain complex of $K / F$ in the case of a rank two slope group. As a more general illustration, we give an example of how one traces back through the attaching maps to find out which cells appear on some geometric face.

We consider the case of a rank three slope group with $n_{1}=2, n_{2}=3$, and $n_{3}=5$. Let $J=\{1,2,3\}$, and consider the geometric $B_{1}$ face of $x_{J}$. It is the union of two-cells in the boundary of the four-cell $x_{J^{\prime}} x_{J^{\prime}}$, where $J^{\prime}=\{2,3\}$. Examining the attaching map for the $B_{1}$ face, we see that it is attached to four squares: $v x_{J^{\prime}}, x_{J^{\prime}} v^{15}$, and certain diagonals of $x_{2} x_{3}^{3}$ and $x_{3} x_{2}^{5}$. But now tracing through the attaching maps for the boundary of $x_{J^{\prime}} x_{J^{\prime}}$, we see that on the $B_{1}$ face we have $v x_{J^{\prime}}, x_{J^{\prime}} v^{15}, x_{2} v^{2} x_{3}, x_{2} v x_{3} v^{5}, x_{2} x_{3} v^{10}, x_{3} v^{4} x_{2}, x_{3} v^{3} x_{2} v^{3}$, $x_{3} v^{2} x_{2} v^{6}, x_{3} v x_{2} v^{9}$, and $x_{3} x_{2} v^{12}$.

Notice in this example that $\sigma=v x_{J^{\prime}}$ is redundant, and $c(\sigma)=x_{J}$. One checks that in general $\sigma$ is on the boundary of $c(\sigma)$. So all that remains to be verified is that there are no infinite chains of immediate predecessors. To do this we will first need to define a partial order on the $\chi(\sigma)$ 's, for $\sigma$ an $m$-cell, as in $\S 2$. First we define a partial order on the letters $x_{J}$, with $J \neq \varnothing$. If $|J|<\left|J^{\prime}\right|$, then we set $x_{J}<x_{J^{\prime}}$, unless $\left|J^{\prime}\right|=|J|+1,1 \in J^{\prime}$, and $1 \notin J$, in which case we set $x_{J}^{\prime}<x_{J}$. We take the transitive closure of this order. Now we again order the $\chi$ 's lexicographically, reading from the right. Again, since the number of $x_{J}$ 's in an $m$-cell is bounded, there are no infinite descending chains of $\chi$ 's for $m$-cells. Now one checks:

Claim 3.6. Suppose $\tau^{\prime}$ is an immediate predecessor of $\tau$. Then either (1) $\chi\left(\tau^{\prime}\right)<\chi(\tau)$, or (2) $\chi(\tau)=\chi\left(\tau^{\prime}\right)$, in which case $\tau^{\prime}=R v^{a} x_{J} v^{b} S$ and $\tau=$ $R v^{c} x_{J} v^{d} S$, where either $a<c$, or $a=c$ and $b<a$.

Notice in the example of the three-cell above that all of the faces listed above other than $\sigma$ and $x_{J^{\prime}} v^{15}$ fall into case (1) of the claim, and $x_{J^{\prime}} v^{15}$ falls into case (2). It follows from the claim that there are no infinite chains of immediate predecessors. So we may collapse $K / F$ to a complex $Y$ with one cell for each essential cell of $K$.

While this is a more complete collapse than the collapse of $\S 2$, the boundary maps in the cellular chain complex of $Y$ are not always zero. In the cases where $d \neq n_{1}-1$, the complex would have more than one vertex, so clearly the boundary maps cannot all be zero. In these cases one could collapse a maximal tree, but it is not clear how to do further collapsing systematically. If $d=n_{1}-1$, the boundary maps are zero. This follows from a homology computation which may be deduced from the work of P. Greenberg [8]. If $k=2$ and $d=r_{1}$, we have checked directly that the boundary maps are zero. In all cases, though, this collapse does provide smaller finite presentations for the groups than the ones obtained in $\S 2$. We give as an example the finite presentation corresponding 
to the infinite presentation of Example 2 of $\S 1$. Notice that in this example $d=n_{1}-1$, so this presentation is the smallest possible one.

Example. For $F\left(1, \mathbb{Z}\left[\frac{1}{6}\right],\langle 2,3\rangle\right)$ we obtain

$$
\begin{array}{r}
\left\langle x_{0}, x_{1}, y_{0}, y_{1}\right| x_{2} x_{0}=x_{0} x_{3}, x_{3} x_{1}=x_{1} x_{4}, y_{2} x_{0}=x_{0} y_{3}, \\
y_{3} y_{1}=y_{1} y_{4}, x_{1} y_{0}^{+}=y_{0}^{+} x_{3}, x_{2} y_{1}^{+}=y_{1}^{+} x_{4}, \\
y_{1} y_{0}^{+}=y_{0}^{+} y_{3}, y_{2} y_{1}^{+}=y_{1}^{+} y_{4}, \\
\left.x_{0} y_{1}^{+} y_{0}^{+}=y_{0}^{+} x_{2} x_{1} x_{0}, x_{1} y_{2}^{+} y_{1}^{+}=y_{1}^{+} x_{3} x_{2} x_{1}\right\rangle,
\end{array}
$$

where $x_{i-2}^{-1} x_{i-1} x_{i-2}=x_{i} \quad \forall i \geq 2, x_{i-2}^{-1} y_{i-1} x_{i-2}=y_{i} \quad \forall i \geq 2$, and $y_{i}^{+}=$ $y_{i+1}^{-1} y_{i} x_{i+1} x_{i} \quad \forall i$.

We would like to be able to compute $H_{*}(F) \cong H_{*}(K / F)$ in any of our examples. If the slope group has rank one, the collapse always gives the answer. If the slope group has rank two, the collapse only gives the answer if $d=n_{1}-1$. In the next section we compute the homology of $K / F$ for any slope group of rank two. We have found the computations too complicated for slope groups of higher rank. To do this computation, we would like a description of the cellular chain complex of $K / F, C(K / F)$.

$K$ is a regular $\mathrm{CW}$-complex with cubes as the cells. Sometimes an $n$-cube has a union of $(n-1)$-cubes as a geometric face, rather than just one $(n-1)$-cube, as in a normal cubical complex. $C^{n}(K / F)$ is a free abelian group with the set of all symbols for $n$-cubes as a basis. Given a cell $\sigma$ of $K$, we can list the cells which appear in $\partial(\sigma)$ by seeing which ones appear in the image of the attaching maps. Since $K$ is a regular CW-complex, each one appears with coefficient \pm 1 , and one only need check that $\partial^{2}=0$ and that the two endpoints of an edge have opposite sign to ensure that one has the correct boundary operator (see [6, Chapter 3]).

One checks that the following boundary formula works:

$$
\partial(\sigma)=\sum_{i=1}^{n}(-1)^{i}\left(A_{i}(\sigma)-B_{i}(\sigma)\right),
$$

where $A_{i}$ and $B_{i}$ are defined as follows. Suppose $\sigma=\operatorname{SmR}$, where $m \in$ $\left\{x_{1}, x_{2}, x_{\{1,2\}}\right\}$ and $S$ is a $(j-1)$-cell. Then if $m=x_{i}$,

$$
A_{j}(\sigma)=S v R, \quad B_{j}(\sigma)=S v^{n_{i}} R
$$

and if $m=x_{\{1,2\}}$, then

$$
\begin{gathered}
A_{j}(\sigma)=S x_{1} R, \quad A_{j+1}(\sigma)=S x_{2} R, \\
B_{j}(\sigma)=S v^{r_{2}} x_{1} R+S v^{r_{2}-1} x_{1} v^{r_{1}+1} S+\cdots+S x_{1} v^{r_{2}\left(r_{1}+1\right)} R, \\
B_{j+1}(\sigma)=S v^{r_{1}} x_{2} R+S v^{r_{1}-1} x_{2} v^{r_{2}+1} R+\cdots+S x_{2} v^{r_{1}\left(r_{2}+1\right)} R .
\end{gathered}
$$

\section{Homology CAlculations}

In this section we compute the homology of any $F$ group with rank two slope group. The homology of $F$ is the homology of the cellular chain complex of $K / F$. We will compute this algebraically, using a product structure on the chain complex. We will first give the argument for the rank one case, using the 
chain complex of $N / F$, which is due to Ken Brown. We will then extend this to the rank two case.

First we explain the product. We first fix a choice of slope group $P$ and a basis for $P,\left\{n_{1}, n_{2}\right\}$. We denote $F\left(l, \mathbb{Z}\left[\frac{1}{n_{1} n_{2}}\right],\left\langle n_{1}, n_{2}\right\rangle\right)$ for $l \in\{1,2, \ldots, d\}$ (or $F\left(l, \mathbb{Z}\left[\frac{1}{n}\right],\langle n\rangle\right)$ in the rank one case), by $F_{l}$. Then we define a map $m: F_{a} \times$ $F_{b} \rightarrow F_{a+b}$. If $g \in F_{a}$ and $h \in F_{b}$, we define

$$
m(g, h)(x)= \begin{cases}g(x) & \text { if } x \in[0, a] \\ h(x-a)+a & \text { if } x \in[a, a+b]\end{cases}
$$

If we denote the complex for $F_{l}$ by $K_{l}$ (we use $N_{l}$ in the rank one case), the map $m$ induces a map $m^{\prime}$ among the vertices of the $K_{l}$, i.e., if $f_{1}:\left[0, t_{1}\right] \rightarrow$ $[0, a]$ and $f_{2}:\left[0, t_{2}\right] \rightarrow[0, b]$, so that $f_{1} \in K_{a}$ and $f_{2} \in K_{b}$, then define $m^{\prime}\left(f_{1}, f_{2}\right):\left[0, t_{1}+t_{2}\right] \rightarrow[0, a+b]$ by

$$
m^{\prime}\left(f_{1}, f_{2}\right)(x)= \begin{cases}f_{1}(x) & \text { if } x \in\left[0, t_{1}\right] \\ f_{2}\left(x-t_{2}\right)+a & \text { if } x \in\left[t_{1}, t_{1}+t_{2}\right]\end{cases}
$$

One checks that this extends in the obvious way to a map from $K_{a} \times K_{b}$ to $K_{a+b}$ in which the product of an $m$-cell and an $n$-cell is an $(m+n)$-cell. This product is also compatible with the group action, in the sense that if $\sigma$ is a cell of $K_{a}, \tau$ is a cell of $K_{b}, g \in F_{a}$, and $h \in F_{b}$, then

$$
m^{\prime}(g \sigma, h \tau)=(m(g, h))\left(m^{\prime}(\sigma, \tau)\right)
$$

This implies that we have an induced product among the quotient spaces $K_{l} / F_{l}$.

These maps are defined among an infinite collection of spaces. For computations it would be helpful to be able to consider only finitely many of them. Recall that if we set $d=\operatorname{gcd}\left(n_{1}-1, n_{2}-1\right)$, then $A / I P \cdot A \simeq \mathbb{Z} / d \mathbb{Z}$. But then A.1 tells us that we may choose for each $l$ a map $g_{l}:[0, l] \rightarrow[0, i]$, where $i \in\{1,2, \ldots, d\}$ and $i \equiv l(\bmod d)$, which induces by conjugation an isomorphism between $F_{l}$ and $F_{i}$. Then this isomorphism induces a homotopy equivalence between $K_{l}$ and $K_{i}$, which induces a homotopy equivalence between $K_{l} / F_{l}$ and $K_{i} / F_{i}$. Notice that in the quotient spaces, these homotopy equivalences are independent of the choice of the $g_{l}$. Using them, our product among all of the quotient spaces becomes a product among the finite set of spaces $K_{1} / F_{1}, \ldots, K_{d} / F_{d}$. It is an associative product with no identity, and extending by linearity gives a product among the chain complexes of the $K_{i} / F_{i}, C\left(K_{i} / F_{i}\right)$. Checking the definitions, we see that this is compatible with the boundary maps in the $C\left(K_{i} / F_{i}\right)$, in the sense that

$$
\partial\left(m^{\prime}(\sigma, \tau)\right)=m^{\prime}(\partial(\sigma), \tau)+(-1)^{\operatorname{dim} \sigma} m^{\prime}(\sigma, \partial(\tau))
$$

We summarize this product in terms of our description of $C\left(K_{i} / F_{i}\right)$. Let $Y=C\left(K_{1} / F_{1}\right) \oplus C\left(K_{2} / F_{2}\right) \oplus \cdots \oplus C\left(K_{d} / F_{d}\right)$. Then $Y$ is a graded ring without identity freely generated by $v, x_{1}, x_{2}$, and $x_{\{1,2\}}=z$; of dimensions $0,1,1$, 
and 2, respectively. $Y$ also has a boundary operator $\partial$ defined by:

$$
\begin{aligned}
\partial(\sigma \tau)= & \partial(\sigma) \tau+(-1)^{(\operatorname{dim} \sigma)} \sigma \partial(\tau), \\
\partial(v)= & 0 \\
\partial\left(x_{1}\right)= & v^{n_{1}}-v, \\
\partial\left(x_{2}\right)= & v^{n_{2}}-v, \\
\partial(z)= & v^{n_{2}-1} x_{1}+v^{n_{2}-2} x_{1} v^{n_{1}}+\cdots+x_{1} v^{\left(n_{2}-1\right) n_{1}}-x_{1} \\
& +x_{2}-v^{n_{1}-1} x_{2}-v^{n_{2}-2} x_{2} v^{n_{2}}-\cdots-x_{2} v^{\left(n_{1}-1\right) n_{2}},
\end{aligned}
$$

where we write the product in $Y$ as multiplication is usually written. Note that in the rank one case, we have a product in the direct sum of the $C\left(N_{i} / F_{i}\right)$, which we will also call $Y$, with the same description except for the fact that we have no $x_{2}$ or $z$ generators.

Now we want to use $Y$ to compute the homology of the $F_{i}$, but $H_{*}(Y)=$ $\bigoplus_{i=1}^{d} H_{*}\left(F_{i}\right)$. This is not a problem, however, because if we let $B_{i}$ be the subgroup of $F_{i}$ consisting of homeomorphisms which are the identity in neighborhoods of 0 and $i$, then $B_{i} \cong B_{j}$ (see A.4), and we have:

Lemma 4.1 (K. S. Brown). $H_{*}\left(F_{i}\right) \cong H_{*}\left(B_{i}\right) \otimes H_{*}(P \times P)$. In particular, $H_{*}\left(F_{i}\right) \cong$ $H_{*}\left(F_{j}\right), i, j \in\{1, \ldots, d\}$.

Proof of 4.1. If we let $\rho: F_{i} \rightarrow P \times P$ be the map which records the slope at the two ends of a homeomorphism, then we have a split exact sequence

$$
0 \rightarrow B_{i} \stackrel{\text { incl. }}{\longrightarrow} F_{i} \stackrel{\rho}{\rightarrow} P \times P \rightarrow 0 .
$$

Then we can form the following commutative diagram:

$$
\begin{array}{ccccccccc}
0 & \rightarrow & B_{i} & \stackrel{\text { incl. }}{\longrightarrow} & F_{i} & \stackrel{\rho}{\rightarrow} & P \times P & \rightarrow & 0 \\
& & \left.\downarrow m\right|_{B_{i}} & & \downarrow(m, \rho) & & \downarrow \text { id } & & \\
0 & \rightarrow & B_{i} & \stackrel{\text { incl. }}{\longrightarrow} & B_{i} \times(P \times P) & \stackrel{\text { proj. }}{\longrightarrow} & P \times P & \rightarrow & 0
\end{array}
$$

where to define $m$ we choose a piecewise linear homeomorphism $\varphi$ with slopes in $P$ and singularities in $A$ taking $[0, i]$ to some proper closed subinterval $\left[0+\varepsilon_{1}, i-\varepsilon_{2}\right]$. If $h \in F_{i}$, let

$$
m(h)(x)= \begin{cases}x, & x \in\left[0, \varepsilon_{1}\right] \cup\left[i-\varepsilon_{2}, i\right], \\ \varphi \circ h \circ \varphi^{-1}, & x \in\left[\varepsilon_{1}, i-\varepsilon_{2}\right] .\end{cases}
$$

Now $\left.m\right|_{B_{i}}$ induces the identity on $H_{*}\left(B_{i}\right)$, for if $z$ is a cycle in the standard resolution for $B_{i}$ representing the homology class $\bar{z}, z$ involves only finitely many elements of $B_{i}$ and the union of the supports of all of these homecmorphisms is contained in some open subinterval of $[0, i]$. So $\exists f \in B_{i}$ such that $f \circ g \circ f^{-1}=\varphi \circ g \circ \varphi^{-1}$ for all $g \in B_{i}$ appearing in $z$. So $\left(\left.m\right|_{B_{i}}\right)_{*}(\bar{z})=f_{*}(\bar{z})$, where $f_{*}$ is the map on homology induced by conjugation by $f$. But $f_{*}=\left.\mathrm{id}\right|_{B_{i}}$, so $\left(\left.m\right|_{B_{i}}\right)_{*}$ is just the identity map. The commutative diagram then induces the identity maps between the $E^{2}$ pages of the spectral sequences for the two exact sequences, so $(m, \rho)$ induces an isomorphism on homology, and

$$
H_{*}\left(F_{i}\right) \cong H_{*}\left(B_{i} \times P \times P\right) \cong H_{*}\left(B_{i}\right) \otimes H_{*}(P \times P) .
$$

Since $B_{i} \cong B_{j}$, this proves the lemma. 
We will now find the homology of $Y$, keeping in mind that it is $d$ copies of the homology of $F_{i}$ for any $i$. We first consider the case of one integer, and compute the homology of $Y$, using an argument of K. S. Brown. Now the slope group $P=\langle n\rangle$, and recall that $d=n-1$. We first make $Y$ into a graded ring $R$ with identity by setting $R=Y \oplus \mathbb{Z}$, where the extra $\mathbb{Z}$ is just generated by the identity for $R$. Consider the following chain subcomplex $C$ of $R: C_{0}$ is generated by $v^{n}-v=w, C_{1}$ is generated by $x, m_{1}=v x-x v$, $m_{2}=v^{2} x-x v^{2}, \ldots, m_{n-1}=v^{n-1} x-x v^{n-1}$. Notice that $\partial\left(m_{i}\right)=0 \forall i$, and $\partial(x)=w$, so that $H_{0}(C)=0$ and $H_{1}(C)=\mathbb{Z}^{d}$. Now given an arbitrary monomial in $R$, i.e., a string of $v$ 's and $x$ 's, we can almost rewrite it as a sum of monomials involving only $x, w$, and $m_{i}$. Start at the left end of the monomial, and leave any $x$ 's that you see alone. If you see a $v$, count the number which occur in a row, say $i$. If $i \geq n$, replace the leftmost $v^{n}$ by $w+v$, which you can think of as reducing the number of $v$ 's. Continue, until you are reduced to the case $i<n$. If this is the end of the word, stop. If you see $v^{i} x$, replace it by $m_{i}+x v^{i}$, which you can think of as moving $v^{i}$ past the $x$. Continue until you have the sum of monomials only in the $m_{i}, w$, and $x$, except possibly with $v^{j}$ at the right ends for $j \in\{1,2, \ldots, d\}$.

Claim 4.2. The monomials described above form a basis for $R$ as an abelian group.

Proof of 4.2. Let $S$ be the free graded ring with identity generated by $w^{\prime}, x^{\prime}$, and $m_{i}^{\prime}$, with gradings $n, n$, and $n+i$, respectively. Let $S v^{i}$, for $i \in$ $\{1, \ldots, d\}$, be an isomorphic copy of $S$, where if $m \in S$ is a monomial of degree $r$, the copy of $m$ in $S v^{i}$ has degree $r+i$. Then $\mathscr{S}=S \oplus S v \oplus S v^{2} \oplus \cdots \oplus$ $S v^{d}$ is a graded ring, and we let $F^{r}(\mathscr{S}) \subset \mathscr{S}$ consist of all sums of monomials of degrees $\leq r$. Map $\mathscr{S}$ to $R$ via a map $\alpha$, where if $m$ is a monomial in $S$, so that $m=m\left(w^{\prime}, x^{\prime}, m_{1}^{\prime}, \ldots, m_{d}^{\prime}\right)$, then $\alpha\left(m\left(w^{\prime}, x^{\prime}, m_{1}^{\prime}, \ldots, m_{d}^{\prime}\right)\right)=$ $m\left(w, x, m_{1}, \ldots, m_{d}\right) \in R$. Extend this to $\mathscr{S}$ by sending the copy of $m$ in $S v^{j}$ to $m\left(w, x, m_{1}, \ldots, m_{d}\right) \cdot v^{j} \in R$. The argument of the previous paragraph shows that this map is surjective. To show that it is injective, we give $R$ a new grading by setting $\operatorname{dim}(v)=1$ and $\operatorname{dim}(x)=n$. We let $F^{r}(R) \subset R$ consist of all sums of monomials of degree $\leq r$. Then our map $\alpha$ takes $F^{r}(\mathscr{S})$ to $F^{r}(R)$, so we have an induced map $\bar{\alpha}$ on the associated graded objects. One easily checks that $\bar{\alpha}$ is still surjective (it differs from $\alpha$ only in that $w$ is now sent to $v^{n}$ instead of $\left.v^{n}-v\right)$. So $\bar{\alpha}$ takes $F^{r}(\mathscr{S}) / F^{r-1}(\mathscr{S})$ onto $F^{r}(R) / F^{r-1}(R)$. But these are both finitely generated free abelian groups, so if they are of the same rank for every $r$, then $\bar{\alpha}$ is an isomorphism. So we count basis elements on both sides, recording the information in power series $P(t)=\sum a_{i} t^{i}$, where $a_{i}$ is the rank of the subgroup of dimension $i$. We have

$$
\begin{aligned}
P_{S}(t)= & 1+\left(2 t^{n}+t^{n+1}+t^{n+2}+\cdots+t^{2 n-1}\right) \\
& +\left(2 t^{n}+t^{n+1}+t^{n+2}+\cdots+t^{2 n-1}\right)^{2}+\cdots \\
= & \frac{1}{1-2 t^{n}-t^{n+1}-t^{n+2}-\cdots-t^{2 n-1}}, \\
P_{\mathscr{S}}(t)= & \frac{1+t+t^{2}+\cdots+t^{d}}{1-2 t^{n}-t^{n+1}-t^{n+2}-\cdots-t^{2 n-1}}, \\
P_{R}(t)= & 1+\left(t+t^{n}\right)+\left(t+t^{n}\right)^{2}+\cdots=\frac{1}{1-t-t^{n}},
\end{aligned}
$$


and we see that $P_{\mathscr{S}}(t)=P_{R}(t)$, so $\bar{\alpha}$ is an isomorphism, which implies that the original map $\alpha$ is also an isomorphism.

We can now think of $R$ as $S \oplus S v \oplus S v^{2} \oplus \cdots \oplus S v^{d}$, where $S \cong \mathbb{Z} \oplus C \oplus$ $(C \otimes C) \oplus(C \otimes C \otimes C) \oplus \cdots$. Now by the Künneth formula, $H_{0}(S)=\mathbb{Z}$ and $H_{k}(S)=\mathbb{Z}^{d^{k}}, k \geq 1$; and so $H_{0}(R)=\mathbb{Z}^{n}$ and $H_{k}(R)=\mathbb{Z}^{n(n-1)^{k}}, k \geq 1$. But remember that we put an extra $\mathbb{Z}$ into dimension zero when changing $Y$ to $R$, and $H_{*}(Y)$ was really $n-1$ copies of the homology of $F_{i}$. So we have algebraically recovered the result of Theorem 2.6.

Remark. For future reference, notice that if we convert some cycle in $R$ to its normal form, meaning a sum of monomials in only $x, w$, and $m_{i}$, except possibly fewer than $n v$ 's at the right, the sum of the terms having at least one $x$ or $w$ must be a boundary in $R$.

Now we would like to do this algebra in the rank two case, to determine the homology of $F_{i}$ in all cases. Again we form $R=Y \oplus \mathbb{Z}$ to give our ring an identity, and again we would like to find a small chain complex $C$ with easily computable homology which freely generates the ring. To do this we need to change the basis for $R$ to make the boundary maps simpler. We begin with the two slope group generators, $n_{1}$ and $n_{2}$, and assume $n_{1}<n_{2}$. Let $r_{1}=n_{1}-1$ and $r_{2}=n_{2}-1$, and perform the euclidean algorithm on $r_{1}$ and $r_{2}$. After the first step we obtain $r_{3}$, with $0 \leq r_{3}<r_{1}$ and $r_{2}=a_{1} r_{1}+r_{3}$. Then we replace $x_{2}$ by $x_{2}-x_{1} v^{r_{1}-r_{2}}-x_{1} v^{r_{1}-2 r_{2}}-\cdots-x_{1} v^{r_{s}}$ in the basis for $R$. Continuing in this manner, performing the euclidean algorithm and making a corresponding change in the basis for $R$, we obtain:

$$
\begin{aligned}
& \begin{array}{ccc}
r_{2}= & a_{1} r_{1}+r_{3} \\
r_{1}= & a_{2} r_{3}+r_{4} \\
\vdots & \vdots & \vdots \\
r_{f-2}= & a_{f-2} r_{f-1}+r_{f} \\
r_{f-1}= & a_{f-1} r_{f},
\end{array} \\
& x_{3}=x_{2}-x_{1} v^{r_{1}-r_{2}}-\cdots-x_{1} v^{r_{3}} \\
& x_{4}=x_{1}-x_{3} v^{r_{2}-r_{3}}-\cdots-x_{3} v^{r_{4}} \\
& \vdots \quad \vdots \quad \vdots \\
& x_{f}=x_{f-2}-x_{f-1} v^{r_{(f-2)}-r_{(f-1)}}-\cdots-x_{f-1} v^{r_{f}} \\
& x_{f+1}=x_{f-1}-x_{f} v^{r_{(f-1)}-r_{f}}-\cdots-x_{f} \text {, }
\end{aligned}
$$

where $r_{f}=d$. We then replace $x_{1}$ and $x_{2}$ by $x_{f}$ and $x_{f+1}$ in our basis for $R$. The advantage to this is that $\partial\left(x_{f+1}\right)=0$ and $\partial\left(x_{f}\right)=v^{d+1}-v$, so that when we add $x_{f+1}$ and $x_{f}$ to $C$, we only have to add one boundary, $v^{d+1}-v$, in order to make the homology of $C$ easy to compute. Our problem now is that $\partial(z)$ is an expression in $x_{1}, x_{2}$, and $v$, and when we rewrite it in terms of the new basis it is quite a bit longer and messier. To handle this, we notice that if $\partial(z)=R_{f}+R_{f+1}$, where $R_{f}$ is the sum of all terms in $x_{f}$ and $v$ and $R_{f+1}$ is the sum of all terms in $x_{f+1}$ and $v$, then $\partial\left(R_{f+1}\right)=0$. But this means that $R_{f}$ is also a cycle. It would be nice if $R_{f}$ were actually a boundary, for then we could change basis again to eliminate it from $\partial(z)$. In fact, this is true.

Proposition 4.3. $R_{f}$ is the boundary of $B_{1}$, where $B_{1}$ is some expression involving only $x_{f}$ and $v$. 
Proof of 4.3. $R_{f}$ is a sum of terms of the form $v^{e} x_{f} v^{h}$. Notice that $R_{f}$ is in the chain complex generated by $v$ and $x_{f}$, with $\partial\left(x_{f}\right)=v^{d+1}-v$. But this is just the chain complex $Y$ for the rank one case with $n=d+1$, which we already understand. Recalling the remark made after the proof of 5.2, if we can show that when we rewrite $R_{f}$ as a sum of strings of $x_{f}, w=v^{d+1}-v$, and $m_{i}=v^{i} x_{f}-x_{f} v^{i}$, with perhaps as many as $d v$ 's on the right, that no terms of the form $m_{i} v^{j}$ appear, then $R_{f}$ is a boundary. So we need to keep track of these terms as they appear as we rewrite $R_{f}$, and check that in the end they all cancel. To see what happens to a typical term $v^{e} x_{f} v^{h}$, let $e \equiv i(\bmod d)$ and $h \equiv j(\bmod d)$, with $i, j \in\{1,2, \ldots, d\}$. Then we see that reducing $v^{e} x_{f} v^{h}$ yeilds:

(i) no $m_{s} v^{p}$ term if $e=0$,

(ii) $m_{i}$ if $e \neq 0$ but $h=0$,

(iii) $m_{i} v^{j}$ if both $e, h>0$.

One can check that the following two claims hold.

Claim 4.4. Rewrite $x_{1}$ and $x_{2}$ in terms of the new basis elements $v, x_{f}$, and $x_{f+1}$. Let

$a=$ number of terms in $x_{2}$ of the form $x_{f+1} v^{i}$,

$b=$ number of terms in $x_{1}$ of the form $x_{f+1} v^{i}$,

$s=$ number of terms in $x_{2}$ of the form $x_{f} v^{i}$,

$p=$ number of terms in $x_{1}$ of the form $x_{f} v^{i}$.

Then $r_{2}=p d, r_{1}=s d$, and $r_{1} a-r_{2} b=d$ if $f$ is even, and $r_{1} a-r_{2} b=-d$ if $f$ is odd.

Claim 4.5. (1) If $f$ is even, $x_{2}$ will have one $x_{f+1} v^{j}$ term and one $x_{f} v^{j}$ term with $j=0$. All other terms have $j>0, j \equiv 0(\bmod ) . x_{1}$ will have one $x_{f} v^{j}$ term with $j=0$, and all other $x_{f+1} v^{j}$ and $x_{f} v^{j}$ terms have $j>0, j \equiv 0$ $(\bmod d)$.

(2) If $f$ is odd, $x_{2}$ has one $x_{f} v^{j}$ term with $j=0$, and all others with $j>0, j \equiv 0(\bmod d) . \quad x_{1}$ has one $x_{f} v^{j}$ term and one $x_{f+1} v^{j}$ term with $j=0$, and the rest with $j>0, j \equiv 0(\bmod d)$.

With the aid of these, we can count the $m_{i} v^{j}$ terms in $R_{f}$. The claims keep track of the number of $v$ 's to the right and the total number of terms, and the formula for $\partial(z)$ tells us which $m_{i}$ appears in each term. If we let $A=m_{d} v^{d}+m_{(d-1)} v+m_{(d-2)} v^{2}+\cdots+m_{1} v^{(d-1)}$, we see that the $x_{f} v^{h}$ terms from $x_{2}$ contribute

$$
s(p-1) A+\left(A-m_{d}+m_{d}\right)+(s-1) A
$$

to $R_{f}$, and the $r_{f}$ terms from $x_{1}$ contribute

$$
-p(s-1) A-\left(A-m_{d}+m_{d}\right)-(p-1) A
$$

to $R_{f}$. We see that they all cancel, which proves 4.3.

We can simplify our formulas still further with:

Proposition 4.6. $R_{f+1}=v^{d} x_{f+1}+v^{d-1} x_{f+1} v+\cdots+x_{f+1} v^{d}-x_{f+1}+\partial\left(B_{2}\right)$, where $B_{2}$ involves only $v, x_{f}$, and $x_{f+1}$.

Proof of 4.6. Notice that

$$
\partial\left(x_{f+1} x_{f}\right)=x_{f+1} v-x_{f+1} v^{(d+1)}
$$


and

$$
\partial\left(x_{f} x_{f+1}\right)=v^{(d+1)} x_{(f+1)}-v x_{f+1} .
$$

We can add boundaries of these two chains, with $v$ 's on the right and left, to reduce $R_{f+1}$ to a sum of terms of the form $v^{i} x_{f+1} v^{j}$, with $i, j \in\{0,1,2 \ldots, d\}$. Using Claim 4.5, we count what is left and see that we have $\pm\left(v^{d} x_{f+1}+\right.$ $\left.v^{d-1} x_{f+1} v+\cdots+x_{f+1} v^{d}-x_{f+1}\right)$, so if we let $B_{2}$ be \pm (the sum of the terms whose boundaries we added), we have the statement of the proposition.

Now we may change our basis for $R$ again by replacing $z$ by $z^{\prime}=z-B_{1}-B_{2}$. So now $R$ is a ring freely generated by $v, x_{f}, x_{f+1}$, and $z^{\prime}$, where $\partial(v)=0$, $\partial\left(x_{f}\right)=v^{d+1}-v, \partial\left(x_{f+1}\right)=0$, and $\partial\left(z^{\prime}\right)=v^{d} x_{f+1}+\cdots+x_{f+1} v^{d}-x_{f+1}$.

Next we need to find cycles to move the $v$ 's to the right of $x_{f}, x_{f+1}$, and $z^{\prime}$. For $x_{f}$ and $x_{f+1}$ this is easy. We let

$$
m_{i}=v^{i} x_{f}-x_{f} v^{i} \text { for } i \in\{1,2, \ldots, d\}
$$

and

$$
y_{j}=v^{j} x_{f+1} \text { for } j \in\{1,2, \ldots, d-1\} .
$$

Note that we do not need $v^{d} x_{f+1}$ to move $d v$ 's past $x_{f+1}$, since $\partial\left(z^{\prime}\right)$ will play this role. Now let

$$
l_{i}=\sum_{k=0}^{i-1} v^{k} x_{f} x_{f+1} v^{i-1-k}+\sum_{k=0}^{i-1} v^{k} x_{f+1} x_{f} v^{i-1-k} .
$$

Then $\partial\left(l_{i}\right)=\partial\left(v^{i} z^{\prime}-z^{\prime} v^{i}\right)$, and we let

$$
c_{i}=v^{i} z^{\prime}-z^{\prime} v^{i}+l_{i} \text { for } i \in\{1,2, \ldots, d\} .
$$

We now make the same argument that we had for the rank one case. Let $C$ be the chain complex generated by one zero-chain $w=v^{d}-v$; the one-chains $x_{f}, x_{f+1}$, the $m_{i}$, the $y_{i}$, and $\partial\left(z^{\prime}\right)$; and the two-chains $z^{\prime}$ and the $c_{i}$. We can now write anything in $R$ as sums of strings of the generators of $C$, except for perhaps $d$ or fewer $v$ 's at the right of the strings. Now we argue exactly as before, making a map from $S \oplus S v \oplus \cdots \oplus S v^{d}$ to $R$, where $S$ is the free ring on the normal forms with no $v$ 's at the right. We give $R$ a new grading with $\operatorname{dim}(v)=1, \operatorname{dim}\left(x_{f}\right)=d+1, \operatorname{dim}\left(x_{f+1}\right)=1$, and $\operatorname{dim}\left(z^{\prime}\right)=d+1$. We can then give $S \oplus S v \oplus \cdots \oplus S v^{d}$ a grading by giving the corresponding generators the same dimensions as above, and setting the dimensions of the generators which are mapped to $m_{i}, y_{i}, c_{i}, w$, and $\partial\left(z^{\prime}\right)$ to be $i+d+1, i+1, d+i+1, d+1$, and $d+1$, respectively. Now our surjective map preserves the filtration given by this grading and, as before, the induced map on the associated graded objects is still surjective. By counting one shows it to be an isomorphism, and our conclusion is

Theorem 4.7. Let $F_{i}=F\left(i, \mathbb{Z}\left[\frac{1}{n_{1} n_{2}}\right],\left\langle n_{1}, n_{2}\right\rangle\right)$, where $n_{1}$ and $n_{2}$ form a basis for $\left\langle n_{1}, n_{2}\right\rangle$. Let $d=\operatorname{gcd}\left(n_{1}-1, n_{2}-1\right)$. Then $H_{j}\left(F_{i}\right)$ is a free abelian group, and if we set $h_{j}\left(F_{i}\right)=\operatorname{rk}_{\mathbb{Z}}\left(H_{j}\left(F_{i}\right)\right)$, then the ranks are given by

$$
h_{0}\left(F_{i}\right)=1, \quad h_{1}\left(F_{i}\right)=2(d+1), \quad h_{2}\left(F_{i}\right)=(1+4 d)(d+1),
$$

and

$$
h_{j}\left(F_{i}\right)=d h_{j-2}\left(F_{i}\right)+2 d h_{j-1}\left(F_{i}\right) \text { for } j>2 \text {. }
$$


Remark. Notice that $\bigoplus_{i=1}^{d} H_{*}\left(F_{i}\right)$, as a ring, is generated by a finite number of elements in dimensions zero and one in the case of a rank one slope group, and zero, one, and two in the case of a rank two slope group. Now by the universal coefficient theorem, computing the cup product in cohomology is equivalent to computing $\Delta_{*}$ on homology, where $\Delta$ is the diagonal map. Since $\Delta_{*}$ is a ring homomorphism, all we need to compute are the images of the generators of $\bigoplus_{i=1}^{d} H_{*}\left(F_{i}\right)$. One can also compute the homology and cohomology of the subgroup $B_{i}$, at least in some examples.

\section{SiMPLICITY RESULTS}

In this section we use methods similar to those of [7], variants of which have been used to establish the simplicity results for $T\left(l, \mathbb{Z}\left[\frac{1}{n}\right],\langle n\rangle\right), G\left(l, \mathbb{Z}\left[\frac{1}{n}\right],\langle n\rangle\right)$, and the general $F$ groups. Recall that the subgroup $B$ of $F$ consisting of all homeomorphisms supported in some proper open subinterval of $[0, l]$ has a simple commutator subgroup, $B^{\prime}$. But from Lemma 4.1 it follews that $B^{\prime}=$ $F^{\prime}$. So $F$ always has a simple commutator subgroup. The examples of $T$ groups with $P$ generated by one integer have been shown to have simple second commutator subgroups [2], and the corresponding examples of $G$ groups have simple commutator subgroups [9]. We complete this with

Theorem 5.1. For any choice of $l, P$, and $A, T(l, A, P)$ has a simple second commutator subgroup, and $G(l, A, P)$ has a simple commutator subgroup.

We will begin by looking at the $T$ groups. Then we have

Theorem 5.2. Define $\nu: T(l, A, P) \rightarrow A /\langle I P \cdot A, l\rangle$ by $\nu(f)=f(a)-a$ for $f \in T$, any $a \in[0, l)$. Then $\nu$ is a well-defined homomorphism, and if $K$ is the kernel of $\nu$, then $K^{\prime}$, the commutator subgroup of $K$, is simple.

We prove this with a series of lemmas.

Lemma 5.3. $\nu$ is a well-defined homomorphism.

Proof of 5.3. Let $a, b \in[0, l) \cap A$ with $a<b$. Then if $f(a)<f(b)$, we have $(f(a)-a)-(f(b)-b) \in I P \cdot A$ [A.1]. If, on the other hand, $f(a)>f(b)$, then $(f(a)-a)-(f(b)-b)-l \in I P \cdot A$, so $\nu$ is well defined. But then clearly it is a homomorphism.

Lemma 5.4. $K=B$, where $B$ is the subgroup of $T$ generated by homeomorphisms which fix some nonempty open arc of their domain pointwise.

Proof of 5.4. Clearly $B \subseteq K$. To see that $K \subseteq B$, factor $f \in K$ into $g \circ f^{\prime}$, where $f^{\prime}$ fixes 0 and $g$ is a rotation by $f(0)=r+n l$ with $r \in I P \cdot A$ and $n \in \mathbb{Z}$. Since rotation by $l$ is just the identity map, $g$ is just rotation by $r$. We only need to check that $f^{\prime} \in B$ and $g \in B$. First we look at $f^{\prime}$. Choose $m, n \in(0, l) \cap A, m<n<l, n>f^{\prime}(m)$. Now by [A.1], $f^{\prime}(m)-m \in I P \cdot A$, so there exists some piecewise linear $h$ with slopes in $P$ and singularities in $A$ taking $[m, n]$ to $\left[f^{\prime}(m), n\right]$. Let

$$
f^{\prime \prime}(x)= \begin{cases}f^{\prime}(x) & \text { if } 0 \leq x \leq m, \\ h(x) & \text { if } m \leq x \leq n, \\ x & \text { if } n \leq x \leq l\end{cases}
$$

Now $f^{\prime \prime} \in B$ and $\left(f^{\prime \prime}\right)^{-1} \circ f^{\prime}=$ id on $[0, m]$, so $f^{\prime} \in B$. Now for $g$, write $r=\sum r_{i},-l<r_{i}<l \quad \forall i, r_{i} \in I P \cdot A$, which we may do since $I P \cdot A$ is dense in 
$A$, which is dense in $\mathbf{R}$. So it suffices to show that $g \in B$, where $g$ is a rotation by $r$ with $0 \leq r<l$. Choose $m, n, p \in(0, l) \cap A$ with $m+n+p+r<l$. Then as before, since $r \in I P \cdot A$, there exist piecewise linear homeomorphisms $h_{1}$ and $h_{2}$ with slopes in $P$ and singularities in $A$ taking $[0, m]$ to $[0, m+r]$ and $[m+n, m+n+r+p]$ to $[m+n+r, m+n+r+p]$, respectively. Let

$$
f^{\prime \prime}(x)= \begin{cases}h_{1}(x) & \text { if } 0 \leq x \leq m, \\ x+r & \text { if } m \leq x \leq m+n, \\ h_{2}(x) & \text { if } m+n \leq x \leq m+n+r+p, \\ x & \text { if } m+n+r+p \leq x \leq l\end{cases}
$$

Now $f^{\prime \prime}$ is clearly in $B$, and $\left(f^{\prime \prime}\right)^{-1} \circ g=$ id on $[m, m+n]$, so belongs to $B$, which implies that $g \in B$. So both factors of $f$ are in $B$, so $f \in B$, which implies that $K \subseteq B$.

Lemma 5.5. For any $\varepsilon>0, B$ is generated by the elements which fix pointwise arcs of length greater than or equal to $l-\varepsilon$.

Proof of 5.5. Suppose $g$ is one of the original generators of $B$. Choose $x \in A$, $x \in U$, where $U$ is an open arc with $\left.g\right|_{U}=\mathrm{id}$. Conjugation $g$ by a rotation by $x$ gives a homeomorphism of $[0, l]$ which is supported in some proper open subinterval. But if this is expressible as a product in the set of elements having support in intervals of length $<\varepsilon$, then so is the original $g$. So we may assume $g$ fixes an arc containing 0 . Suppose $g=$ id outside of $(a, b)$, with $b-a>\varepsilon$. First we consider the case where $g\left(a+\frac{\varepsilon}{2}\right)<a+\varepsilon$. Then $\exists x \in\left(a+\frac{\varepsilon}{2}, a+\varepsilon\right) \cap A$ with $g(x)<a+\varepsilon$. As before, we can find a piecewise linear homeomorphism $h$ with slopes in $P$ and singularities in $A$ taking $[x, a+\varepsilon]$ to $[g(x), a+\varepsilon]$. Let

$$
f(y)= \begin{cases}g(y) & \text { if } 0 \leq y \leq x, \\ h(y) & \text { if } x \leq y \leq a+\varepsilon, \\ y & \text { if } a+\varepsilon \leq y \leq l\end{cases}
$$

Now $f$ has support on $(a, a+\varepsilon)$, so it is one of our prospective generators. Now $f^{-1} \circ g$ is supported in $(x, b)$, and $|b-x|=|b-a|-|x-a| \leq|b-a|-\frac{\varepsilon}{2}$, so we have reduced the length of the arc in which $g$ was supported by at least $\frac{\varepsilon}{2}$, which proves the lemma in this case. But if $g\left(a+\frac{\varepsilon}{2}\right) \geq a+\varepsilon$, then $g^{-1}(a+\varepsilon) \leq a+\frac{\varepsilon}{2}$, which implies that $g^{\prime}\left(a+\frac{\varepsilon}{2}\right)<a+\varepsilon$. So apply the above argument to $g^{-1}$.

Lemma 5.6. Suppose $N$ is a nontrivial subgroup of $T$ normalized by $B^{\prime}$, the commutator subgroup of $B$. Suppose $f, g \in T$ and there exist some nonempty open arc on the circle on which both $f$ and $g$ are the identity. Then $[f, g]=$ $f g f^{-1} g^{-1} \in N$.

Proof of 5.6. Throughout this proof we use the fact that given two open arcs $U$ and $V$ such that $U \cup V$ is not the whole circle, we can find a homeomorphism $b \in T$ such that $b$ has support in some proper open subarc of the circle and $b(U) \subseteq V$. Let $V$ be some open arc $\neq$ the whole circle such that $V \supset$ $\operatorname{supp}(f) \cup \operatorname{supp}(g)$, where $\operatorname{supp}(f)$ denotes the support of $f$, or the smallest open arc outside of which $f$ is the identity. Choose $r_{1}, r_{2} \in B$ such that $r_{1}(V), r_{2}(V)$, and $V$ are pairwise disjoint and $V \cup r_{1}(V) \cup r_{2}(V)$ is not all of the circle. Then if we set $f^{\prime}=\left[f, r_{1}\right]$ and $g^{\prime}=\left[g, r_{2}\right], f^{\prime}, g^{\prime} \in B^{\prime}$ and 
$[f, g]=\left[f^{\prime}, g^{\prime}\right]$. Choose an open arc $V^{\prime}$ which is not the whole circle, such that $\operatorname{supp}\left(f^{\prime}\right) \cup \operatorname{supp}\left(g^{\prime}\right) \subset V^{\prime}$. Now suppose $h \in N, h \neq 1$. So $h(x) \neq x$ for some $x$. Choose some open arc $P \ni x$ such that $h(P) \cap P=\varnothing$ and $P \cup V^{\prime}$ is not the whole circle.

Now choose $m \in B$ such that $m(P) \supset V^{\prime}$. $m$ can be chosen to have support in a proper open arc since $P \cup V^{\prime}$ is not the whole circle. We would like $m$ to be in $B^{\prime}$. We can arrange this by choosing $r$ such that $r(\operatorname{supp}(m) \cup P) \cap$ $(\operatorname{supp}(m) \cup P)=\varnothing, r \in B$. Then $m^{\prime}=m r m^{-1} r^{-1}$ also takes $P$ into something containing $V^{\prime}$, and $m^{\prime} \in B^{\prime}$. The point of this is that now we can conjugate $h$ by $m^{\prime}$ to obtain

$$
h^{\prime}=m^{\prime} h m^{\prime-1} \in N \quad \text { and } \quad h^{\prime}\left(V^{\prime}\right) \cap V^{\prime} \neq \varnothing .
$$

But this means that

$$
\left[f^{\prime}, h^{\prime}\right]=\left(f^{\prime} h^{\prime} f^{\prime-1}\right) \circ h^{\prime-1} \in N \Rightarrow\left[\left[f^{\prime}, h^{\prime}\right], g^{\prime}\right] \in N .
$$

But

$$
\text { [ } \left.\left[f^{\prime}, h^{\prime}\right], g^{\prime}\right]=\left[f^{\prime} \circ\left(h^{\prime}\left(f^{\prime}\right)^{-1}\left(h^{\prime}\right)^{-1}\right), g^{\prime}\right]=\left[f^{\prime}, g^{\prime}\right]
$$

since $\operatorname{supp}\left(h^{\prime}\left(f^{\prime}\right)^{-1}\left(h^{\prime}\right)^{-1}\right)=h^{\prime}\left(\operatorname{supp}\left(f^{\prime-1}\right)\right)$ is disjoint from $V^{\prime}$, on which $f^{\prime}$ and $g^{\prime}$ are supported. We had at the very beginning that $\left[f^{\prime}, g^{\prime}\right]=[f, g]$, so $[f, g] \in N$, as claimed.

Now we are ready for

Proof of 5.2. Choose $\varepsilon<\frac{l}{2}$. By Lemma 5.5, the set of all elements of $T$ which are supported in some arc of length $\varepsilon$ generates $B$. By Lemma 5.6, $\left[g_{\alpha}, g_{\beta}\right] \in N$ for $g_{\alpha}, g_{\beta}$ any two of these generators. Now if $g \in B$, then

$$
g\left[g_{\alpha}, g_{\beta}\right] g^{-1}=\left[g g_{\alpha} g^{-1}, g g_{\beta} g^{-1}\right] .
$$

But the hypotheses of Lemma 5.6 are satisfied for $g g_{\alpha} g^{-1}$ and $g g_{\beta} g^{-1}$ since they are satisfied by $g_{\alpha}$ and $g_{\beta}$, so $\left[g g_{\alpha} g^{-1}, g g_{\beta} g^{-1}\right] \in N$, and so all $B$ conjugates of commutators of a $B$-generating set are in $N$, which implies that $B^{\prime} \subseteq N$. In particular, if we take $N \subseteq B^{\prime}$, we see that $B^{\prime}$ is simple. But $B=K$, so $K^{\prime}$ is simple.

Now $T / K$ is abelian, so $T^{\prime} \subseteq K$, which implies that $T^{\prime \prime} \subseteq K^{\prime}$. But $K^{\prime}$ is simple, which means that $T^{\prime \prime}=K^{\prime}$, so $T^{\prime \prime}$ is simple as claimed in Theorem 5.1 .

We now turn to the $G$ groups. Here we do not have to pass to a subgroup; $G$ itself is generated by elements with very small support.

Lemma 5.7. For any $\varepsilon>0, G$ is generated by elements which are the identity except on some interval of length $<\varepsilon$.

Proof of 5.7. Given $h \in G$, we may factor $h$ into $h^{\prime} \circ g$, where $h^{\prime}$ is order preserving on $[0, l)$, and $g$ has slope one everywhere. Then $h^{\prime}$ is a product of our prospective generators by 5.5. Now consider $g$, and let $a$ be the smallest number, possibly 0 , such that $g(a) \neq a$. Choose an interval $I$ such that $\frac{\varepsilon}{4}<|I|<\frac{\varepsilon}{2}$, with $I=[a, a+|I|)$. If $g(I)=I$, then we let

$$
f(x)= \begin{cases}g(x) & \text { if } x \in I, \\ x & \text { if } x \notin I .\end{cases}
$$


Then $f$ is one of our prospective generators, and $g \circ f^{-1}$ still has slope one everywhere, and the smallest number $a^{\prime}$ such that $g \circ f^{-1}\left(a^{\prime}\right) \neq a^{\prime}$ is at least $a+\frac{\varepsilon}{4}$.

If $g(I) \neq I$, we reduce to the case above as follows. We may subdivide $I$ into $n$ intervals $I_{1}, I_{2}, \ldots, I_{n}$, open on the right and closed on the left such that $g$ is linear on $I_{i}$ for all $i$. Let $\sigma$ be the permutation on $n$ letters such that $\left\{x \in g\left(I_{\sigma(1)}\right)\right\}$ are all $<\left\{x \in g\left(I_{\sigma(2)}\right)\right\}$ are all $<\cdots$ are all $<\left\{x \in g\left(I_{\sigma(n)}\right)\right\}$. Now for $b \in A$, using

$$
m_{b}(x)= \begin{cases}x & \text { if } 0 \leq x<a \\ x+b & \text { if } a \leq x<a+|I|, \\ x-|I| & \text { if } a+|I| \leq x<a+|I|+b, \\ x & \text { if } a+|I|+b \leq x<l,\end{cases}
$$

we can move $I$ to the right by $b$. If we choose $b<\frac{\varepsilon}{2}$, then $m_{b}$ will be one of our prospective generators. If we compose $m_{b}$ with a map which moves $m_{b}(I)$ to the right, and so on, we can make a composite of our generators, $m^{1}$, taking the leftmost point of $I$ to the leftmost point of $g\left(I_{\sigma(1)}\right)$. Now if we let $a_{i}$ be the right endpoint of $g\left(I_{\sigma(i-1)}\right)$ for $1 \leq i<n$, we similarly form $m^{i}$ to translate $\left[a_{i}, a_{i}+|I|-\left|I_{\sigma(1)}\right|-\cdots-\left|I_{\sigma(i-1)}\right|\right)$ to the right until $a_{i}$ hits the left-hand endpoint of $g\left(I_{\sigma(i)}\right)$, fixing $\left[0, a_{i}\right)$. If we set

$$
g^{\prime}=\left(m^{n} \circ m^{n-1} \circ \cdots \circ m^{1}\right)^{-1} \circ g,
$$

then $g^{\prime}$ takes $I$ to $I$, has slope one everywhere, and is still the identity on $[0, a)$.

Now we can mimic the proof of Lemma 5.6 and Theorem 5.2 to prove the following theorem, which completes the proof of Theorem 5.1.

Theorem 5.8. Any nontrivial subgroup $N$ of $G$ which is normalized by $G^{\prime}$ contains $G^{\prime}$. In particular, $G^{\prime}$ is simple.

\section{Abelianization of $G$ GROUPS}

In [9], Higman shows that the abelianization of $G_{n, r}$ is either trivial or $\mathbb{Z} / 2 \mathbb{Z}$. Recall that $G_{n, r}=G\left(r, \mathbb{Z}\left[\frac{1}{n}\right],\langle n\rangle\right)$. In this section we will use the complex $N$ on which the groups $G\left(l, \mathbb{Z}\left[\frac{1}{n_{1} n_{2} \cdots n_{k}}\right],\left\langle n_{1}, n_{2}, \ldots, n_{k}\right\rangle\right)$ act to compute the abelianization of any of these groups. In particular, we show that it is always finite, which shows that $G^{\prime}$ is always an infinite simple group which is finitely presented and of type $F P_{\infty}$.

In analyzing $G_{\mathrm{ab}}$, we will want to consider the subgroup $\Pi$ generated by the stabilizers of the bases. These basis stabilizers are finite symmetric groups, so $\Pi$ is generated by elements of finite order. Now $G^{\prime}$ is simple, so $\Pi^{\prime}=G^{\prime}$, and we have

$$
G^{\prime}=\Pi^{\prime} \hookrightarrow \Pi \hookrightarrow G .
$$

So if we can show that $\Pi / \Pi^{\prime}$ and $G / \Pi$ are finite, and compute them, we will know the size of $G / G^{\prime}$. We will actually want an explicit abelianization map, which we will construct by combining one measuring $G / \Pi$ and one measuring $\Pi / \Pi^{\prime}$. First we consider $\Pi / \Pi^{\prime}$. 
Lemma 6.1. $\left|\Pi / \Pi^{\prime}\right| \leq 2$.

Proof of 6.1. Suppose $\left|\Pi / \Pi^{\prime}\right| \neq 1$, and suppose $g$ and $g^{\prime}$ are two generators of $\Pi$ not in $\Pi^{\prime}$. Choose two bases $v$ and $v^{\prime}$ such that $g(v)=v$ and $g^{\prime}\left(v^{\prime}\right)=v^{\prime}$. We can expand $v$ to another basis fixed by $g$ as long as we do the same expansions to each $g$-orbit of $v$-intervals. Taking suitable expansions, we may assume that $v$ and $v^{\prime}$ are expansions of the standard basis. Taking further expansions if necessary, we may assume that $v$ has two intervals $i_{1}$ and $i_{2}$ whose images in $[0, l)$ are disjoint from the images of two $v^{\prime}$ intervals $i_{1}^{\prime}$ and $i_{2}^{\prime}$. Now let $f$ (respectively $f^{\prime}$ ) be the element of $G$ which just interchanges $i_{1}$ and $i_{2}$ (respectively $i_{1}^{\prime}$ and $i_{2}^{\prime}$ ). Then $g=f$ and $g^{\prime}=f^{\prime}$ in $\Pi / \Pi^{\prime}$. Now choose a common expansion of $v$ and $v^{\prime}, w$, which is fixed by $f$ and $f^{\prime}$. This is possible because of the disjointness of the images of the intervals which were moved by $f$ and $f^{\prime}$. But now $f$ and $f^{\prime}$ are in the same copy of a finite symmetric group in $\Pi$, so they are equal in $\Pi / \Pi^{\prime}$.

We now need to know when $\left|\Pi / \Pi^{\prime}\right|$ is one and when it is two. Here we follow Higman's setup from [9]. Suppose that one of the $\left\{n_{1}, n_{2}, \ldots, n_{k}\right\}$ is even, say $n_{i}$. Then if $g \in \operatorname{Stab}(v), g$ is also in $\operatorname{Stab}\left(v^{\prime}\right)$, where $v^{\prime}$ is obtained from $v$ by expanding each $v$-interval into $n_{i}$ equal pieces. But then $g$, viewed as a permutation of the $v^{\prime}$-intervals has even parity. So $g \in \Pi^{\prime}$, and hence $\left|\Pi / \Pi^{\prime}\right|=1$. If all of the integers are odd, we cannot do this trick, and it turns out that in these cases $\left|\Pi / \Pi^{\prime}\right|$ is always 2 . To see this, we will map $G$ onto $\mathbb{Z} / 2 \mathbb{Z}$ in the following way. Given $g \in G$, choose any basis $v$ such that $v$ and $g(v)$ are both expansions of the standard basis. Then $g$ may be factored into $g_{v}^{\prime} \circ p_{v}$, where $p_{v} \in \operatorname{Stab}(v)$ and $g_{v}^{\prime}$ is continuous on $[0, l)$. If we view $p_{v}$ as a permutation of the $v$-intervals, since all $n_{i}$ are odd the parity of $p_{v}$ is independent of the choice of $v$. Now we define $m: G \rightarrow \mathbb{Z} / 2 \mathbb{Z}$ by

$$
m(g)= \begin{cases}\text { parity of } p_{v} & \text { if all } n_{i} \text { are odd } \\ 0 & \text { if at least one } n_{i} \text { is even. }\end{cases}
$$

Proposition 6.2. $\operatorname{Ker}(m) \cap \Pi=\Pi^{\prime}$.

Proof of 6.2. From the discussion above this is clear if one $n_{i}$ is even. If all $n_{i}$ are odd, then given $g \in G$, we can give a set of points $0=b_{0}, b_{1}, \ldots, b_{n}, b_{n+1}$ $=l$ in $A \cap[0, l]$ such that $g$ has constant slope $p_{i}$ on $\left[b_{i}, b_{i+1}\right)$. We set

$$
\pi(g)=\sum_{i=0}^{n} \overline{b_{i+1}-b_{i}} \otimes p_{i},
$$

where $\bar{a}$ for $a \in A$ denotes the coset of $a$ in $A / I P \cdot A$. This is clearly independent of the choice of the $b_{i}$ 's. Note that $g\left(b_{i+1}\right)-g\left(b_{i}\right)=p_{i}\left(b_{i+1}-b_{i}\right) \equiv$ $\left(b_{i+1}-b_{i}\right) \bmod I P \cdot A$. It follows easily that $\pi$ is a homomorphism.

Notice that the map $\pi$ is defined for any $G(l, A, P)$, but we only know how to use it when the slope group is generated by integers. In this situation, recall that $A / I P \cdot A \cong \mathbb{Z} / d \mathbb{Z}$, where $d=\operatorname{gcd}\left(n_{1}-1, n_{2}-1, \ldots, n_{k}-1\right)$, so that

$$
A / I P \cdot A \otimes P \cong \mathbb{Z} / d \mathbb{Z} \otimes \mathbb{Z}^{k} \cong(\mathbb{Z} / d \mathbb{Z})^{k} .
$$

Also, by the usual arguments, if $g \in \operatorname{Stab}(v)$ for some basis $v$, we may assume that $v$ is an expansion of the standard basis. Then $g$ just permutes a subdivision of $[0, l)$, so $\pi(g)=0$, and $\Pi \subseteq \operatorname{Ker}(\pi)$. We will show 
Theorem 6.3. $\Pi=\operatorname{Ker}(\pi)$.

This will follow from the following two propositions.

Proposition 6.4. $G / \Pi$ is generated by $k-1$ elements, and $G / \Pi$ has exponent dividing $d$.

Proposition 6.5. The image of $\pi$ is isomorphic to $(\mathbb{Z} / d \mathbb{Z})^{k-1}$.

Since $\Pi \subseteq \operatorname{Ker}(\pi)$, these two propositions establish the theorem as claimed. The following then gives the abelianization of $G$.

Theorem 6.6. There is an exact sequence

$$
1 \rightarrow G^{\prime} \rightarrow G \stackrel{\pi \oplus m}{\longrightarrow}(\mathbb{Z} / d \mathbb{Z})^{k} \oplus(\mathbb{Z} / 2 \mathbb{Z}),
$$

where $\pi$ has image $(\mathbb{Z} / d \mathbb{Z})^{k-1}$, and $m$ has image $\mathbb{Z} / 2 \mathbb{Z}$ if all generators of the slope group are odd and trivial image if at least one slope group generator is even.

Proof of 6.6. The fact that $\operatorname{Ker}(\pi \oplus m)=\Pi^{\prime}=G^{\prime}$ follows from 6.2 and 6.3. The calculation of the image follows from the definition of $m$ and from 6.5.

The rest of this section will be devoted to the proofs of 6.4 and 6.5. To prove 6.4 , we first need to obtain a generating set for $G / \Pi$. The vertices and onecubes of $N$ form a connected graph on which $G$ acts. So a generating set for $G$ is obtained by choosing a maximal tree in $N / G$ and a lift of it to $N$. Then each edge $e$ of $N / G$ may be lifted to an edge which has one endpoint on the tree. The other endpoint, $v$, of this edge is in the $G$-orbit of a unique vertex of the tree, say $v^{\prime}$. Then we may choose a group element $g_{e}$ taking $v^{\prime}$ to $v$. The set of these $g_{e}$ and the stabilizers of vertices of the tree together generate $G$, so the $g_{e}$ generate $G / \Pi$. We will then use loops in the one-skeleton of $N$ to obtain enough relations among these generators to establish 6.4. Having a basis of size one on the tree complicates the relations we need to consider. This can be avoided by considering $N_{h}$, the subcomplex of $N$ spanned by all bases of size $\geq h$, where $h>l$. $N_{h}$ is still a contractible $G$-complex, and we will use it instead of $N$.

We will now describe a maximal tree in $N_{h} / G . N_{h} / G$ has one vertex corresponding to the set of all bases of size $x, x \equiv l(\bmod d), x \geq h$. We will call this vertex $x$. As before, we let $r_{i}=n_{i}-1$. Let $d_{i}=\operatorname{gcd}\left(d_{i-1}, r_{i}\right)$ with $d_{i-1}=b_{i} d_{i}$ for $2 \leq i \leq k$, where $d_{1}=r_{1}$.

Now given a number $x \geq h, x \equiv l(\bmod d)$, then $x-h$ can be written uniquely as $c_{k}(x) r_{k}+c_{k-1}(x) r_{k-1}+\cdots+c_{1}(x) r_{1}$, with $0 \leq c_{i} \leq b_{i}-1$ for $i>1$. Make a path in $N_{h} / G$ from $h$ to $x$ by traversing $c_{k}(x)$ edges corresponding to simple expansions by $n_{k}$, then $c_{k-1}(x)$ edges corresponding to simple expansions by $n_{k-1}$, etc. At the end, if $c_{1}(x)<0$, we traverse edges in a negative direction, decreasing the size of the endpoints as we move along the path. This gives a unique path from $b$ to $x$. Moreover, if this path passes through $y$ on the way from $h$ to $x$, then $y \geq h, y \equiv l(\bmod d)$. Furthermore, the initial part of this path, from $h$ to $y$, is the unique path we have assigned to $y$. So we have described a tree as desired.

We think of the $k$-tuple $\left(c_{1}(x), c_{2}(x), \ldots, c_{k}(x)\right)$ as labelling the vertex $x$. This notation keeps track of the path along the tree from $h$ to $x$ as well as just 
the number $x$. We can think of the tree inductively as $b_{k}$ smaller trees, one for each of the groups

$$
G_{b+y r_{k}}^{\prime}=G\left(b+y r_{k}, \mathbb{Z}\left[\frac{1}{n_{1} \cdots n_{k-1}}\right],\left\langle n_{1}, \ldots, n_{k-1}\right\rangle\right),
$$

with $0 \leq y \leq b_{k}-1$ (using only bases of size $\geq h$ for each group), joined in a row by edges corresponding to simple $n_{k}$-expansions. Then vertices in the $y$ th subtree are the ones labelled $\left(c_{1}, \ldots, c_{k-1}, y\right)$.

We now turn to the proof of 6.4. Now that we have a tree, a generating set for $G / \Pi$ consists of one generator corresponding to each edge not on the tree. Out of each vertex on the tree, we have one edge corresponding to each integer $n_{1}, n_{2}, \ldots, n_{k}$. An $n_{i}$ edge out of a vertex $x$ gives a generator $g_{i}(x)$. In order to choose specific homeomorphisms realizing this abstract generating set, we need to choose a lift of our tree. We lift our tree to $N_{h}$ by lifting the vertex $h$ to some basis of size $h$ which is an expansion of the standard basis. We call the unique basis of size $x$ on this lifted tree $v_{x}$. Then $g_{i}(x)$ can be taken to be a homeomorphism of $[0, l)$ which takes the basis $v_{x+r_{i}}$ to a basis obtained from $v_{x}$ by expanding one interval into $n_{i}$ equal pieces. Since all choices are equivalent $\bmod \Pi$, we write $g_{i}(x)$ for this $\Pi$-coset. Edges on the tree just give the identity as a generator. In particular, notice that all $g_{1}(x)$ are trivial. We will write

$$
g_{i}(x)=g_{i}\left(c_{1}(x), c_{2}(x), \ldots, c_{k}(x)\right) .
$$

To obtain relations among these generators we will use loops which bound two-cubes of $N$. Recall from $\S 1$ that these squares do not always have onecubes for edges; sometimes the edges are the diagonals of higher dimensional cubes. But then we can just choose a path from one endpoint of the edge to the other which contains only one-cubes. There are two basic types of two-cells in $N_{h} / G$ which yield relations among the generators. The simple type of square corresponds to dividing one interval of a basis of size $x$ into $n_{i}$ equal pieces, and another into $n_{j}$, where $i \neq j$. We draw

$$
\begin{array}{ccc}
x & - & x+r_{j} \\
\mid & & \mid \\
x+r_{i} & - & x+r_{i}+r_{j}
\end{array}
$$

to keep track of this. The relation given by this square is

$$
g_{i}(x) g_{j}\left(x+r_{i}\right)=g_{j}(x) g_{i}\left(x+r_{j}\right) .
$$

The complex type of square (complex because the boundary of the square we draw is not really the loop we use) corresponds to dividing one interval of a basis of size $x$ into $n_{i} n_{j}$ equal pieces, where $i \neq j$. Here we draw

$$
\begin{array}{ccc}
x & - & x+r_{i} \\
\mid & & \mid \\
x+r_{j} & - & x+n_{i} n_{j}-1
\end{array}
$$

To obtain a loop traversing only one-cubes of $N_{h}$, we must replace the righthand vertical edge by $n_{i}$ edges, each corresponding to dividing one interval of the appropriately sized basis into $n_{j}$ pieces. Similarly, the lower horizontal edge is replaced by $n_{j}$ edges each corresponding to a simple $n_{i}$-expansion. 
The relation $\bmod \Pi$ which this loop yields is independent of the choice of replacement edges. It is

$$
g_{i}(x) \underbrace{g_{j}\left(x+r_{i}\right) \cdots g_{j}\left(x+r_{i}+r_{i} r_{j}\right)}_{n_{i} \text { terms }}=g_{j}(x) \underbrace{g_{i}\left(x+r_{j}\right) \cdots g_{i}\left(x+r_{j}+r_{j} r_{i}\right)}_{n_{j} \text { terms }} .
$$

We will now examine enough of these relations to prove the following more explicit form of Proposition 6.4.

Lemma 6.7. $G / \Pi$ is generated by

$$
\begin{aligned}
g_{2} & =g_{2}\left(0, b_{2}-1,0, \ldots, 0\right), \\
g_{3} & =g_{3}\left(0,0, b_{3}-1,0, \ldots, 0\right), \\
& \vdots \\
g_{k} & =g_{k}(\underbrace{0, \ldots, 0}_{k-1}, b_{k}-1) .
\end{aligned}
$$

Furthermore, $G / \Pi$ has exponent dividing $d$.

We first prove two subsidiary lemmas.

Lemma 6.8. For any $j<k$,

$$
g_{k}\left(0, \ldots, 0, c_{j}, c_{j+1}, \ldots, c_{k}\right)=g_{k}\left(0, \ldots, 0, c_{j}^{\prime}, c_{j+1}, \ldots, c_{k}\right)
$$

in $G / \Pi$, provided that either $c_{k} \neq b_{k}-1$ or $j=1$.

Proof of 6.8. If $b_{j}-1=0$, then $c_{j}=c_{j}^{\prime}=0$, so we are done. So assume either $j=1$ or $b_{j}-1>0$, in which case we assume that $c_{j} \neq b_{j}-1$. Look at the following simple square:

$$
\begin{array}{ccc}
x & - & x+r_{k} \\
\mid & & \mid \\
x+r_{j} & - & x+r_{k}+r_{j}
\end{array}
$$

where $x=h+c_{j} r_{j}+\cdots+c_{k} r_{k}$. Since either $c_{k} \neq b_{k}-1$ or $j=1$, both vertical edges correspond to edges on the tree, and so contribute no generators to the relation. This implies that

$$
g_{k}\left(0,0, \ldots, 0, c_{j}, c_{j+1}, \ldots, c_{k}\right)=g_{k}\left(0,0, \ldots, 0, c_{j}+1, c_{j+1}, \ldots, c_{k}\right)
$$

in $G / \Pi$. The proof is completed by allowing $c_{j}$ to range over all possible values except $b_{j}-1$.

Lemma 6.9. Any $g_{k}(x)$ generator with $c_{k} \neq b_{k}-1$ is trivial in $G / \Pi$.

Proof of 6.9. By repeated applications of Lemma 6.8,

$$
\begin{aligned}
g_{k}\left(c_{1}, c_{2}, \ldots, c_{k}\right) & =g_{k}\left(0, c_{2}, c_{3}, \ldots, c_{k}\right) \\
& =g_{k}\left(0,0, c_{3}, \ldots, c_{k}\right) \\
& \vdots \\
& =g_{k}\left(0, \ldots, 0, c_{k}\right),
\end{aligned}
$$

where equality denotes equality in $G / \Pi$. But since $c_{k} \neq b_{k}-1$, this last generator is trivial, which proves the lemma. 
Proof of 6.7. We prove this by induction on $k$. If $k=1$, we have no edges off the tree, and the proposition is true. Suppose it is true for any group with slope group of rank less than $k$. We have seen that our tree is made up of $b_{k}$ smaller trees corresponding to groups with $P=\left\langle n_{1}, \ldots, n_{k-1}\right\rangle$. So by the induction hypothesis, the only $g_{i}(x)$ generators with $2 \leq i \leq k-1$ we need are $g_{i}\left(0, \ldots, b_{i}-1,0, \ldots, 0, y\right)$, with $0 \leq y \leq b_{k}-1$, and they all have exponent $d_{k-1}$. Now for $y<b_{k}-1$, we have the simple relation

$$
\begin{aligned}
& g_{k}\left(0, \ldots, 0, b_{i}-1,0, \ldots, 0, y\right) g_{i}\left(0, \ldots, 0, b_{i}-1,0, \ldots, 0, y+1\right) \\
& \quad=g_{i}\left(0, \ldots, 0, b_{i}-1,0, \ldots, 0, y\right) g_{k}\left(c_{1}, \ldots, c_{i-1}, 0, \ldots, 0, y\right) .
\end{aligned}
$$

But Lemma 6.9 says that both of these $g_{k}$ generators are trivial. So out of all the $g_{i}$ generators with $2 \leq i \leq k-1$, we only need the ones with $y=0$, which are $g_{1}, g_{2}, \ldots, g_{k-1}$. Now Lemma 6.9 shows that the only nontrivial $g_{k}(x)$ generators have $c_{k}(x)=b_{k}-1$. But more simple relations show that

$$
g_{k}\left(c_{1}, c_{2}, \ldots, c_{k-1}, b_{k}-1\right)=g_{k}\left(c_{1}^{\prime}, \ldots, c_{k-1}^{\prime}, b_{k}-1\right) V,
$$

where $V$ is some expression in $g_{2}, \ldots, g_{k-1}$. So we actually only need one $g_{k}(x)$ generator, $g_{k}$. This proves the first part of the proposition.

To prove the second assertion, we consider the complex square

$$
\begin{array}{ccc}
x & - & x+r_{k} \\
\mid & & \mid \\
x+r_{1} & - & x-1+n_{1} n_{k}
\end{array}
$$

where $x=h+\left(b_{k}-1\right) r_{k}$. This yields the relation $g_{k}^{r_{1} / b_{k}} X=1$, where $X$ is some expression in $g_{2}, g_{3}, \ldots, g_{k-1}$. We may commute the generators in this relation since we know that $G / \Pi$ is abelian. Now there is some periodicity in $X$. If we set $m=r_{1} / d_{k-1}$, we see that the first $g_{k}$ generator which appears is $g_{k}\left(1,0, \ldots, 0, b_{k}-1\right)$, and after $m b_{k}$ edges we see $g_{k}\left(1+r_{k} / d, 0, \ldots, 0, b_{k}-1\right)$. But Lemma 6.8 says that these two generators are the same in $G / \Pi$. So in fact $X=Y^{d}$, where $Y$ is an expression in $g_{2}, \ldots, g_{k-1}$. So our relation yields

$$
g_{k}^{r_{1}} Y^{d b_{k}}=g_{k}^{r_{1}} Y^{d_{k-1}}=1 .
$$

But by the induction hypothesis, $Y^{d_{k-1}}=1$, so $g_{k}$ has order dividing $r_{1}$, and so the group has exponent dividing $r_{1}$. Now notice that we could have permuted our $k$ integers before constructing our tree, and could have deduced that the group had exponent dividing $r_{i}$ for any $i$. So the group has exponent dividing $d=\operatorname{gcd}\left(r_{1}, \ldots, r_{k}\right)$, which completes the proof.

Now we can prove 6.5 .

Proof of 6.5. Since we know that $G / \Pi$ is generated by $g_{2}, \ldots, g_{k}$ and $\Pi \subset$ $\operatorname{Ker}(\pi)$, we only need to compute $\pi\left(g_{i}\right)$ for $2 \leq i \leq k$ to determine the image of $\pi$. Recall that $g_{i}$ takes the basis $v_{h+b_{i} r_{i}}$, which we will call $v_{i}$, to a basis $v_{i}^{\prime}$. $v_{i}^{\prime}$ is obtained from $v_{h+\left(b_{i}-1\right) r_{i}}$ by expanding one interval into $n_{i}$ pieces. It is easy to compute $\pi\left(g_{i}\right)$ if $v_{i}$ and $v_{i}^{\prime}$ are both expansions of the standard basis. This will be true unless $c_{1}\left(v_{i}\right)<0$. But in this case we can expand both $v_{i}$ and $v_{i}^{\prime}$ by $n_{1}$ until they are expansions of the standard basis. Now since 
expansions of the standard basis are just $P$-regular subdivisions, which have intervals of length $\overline{1}$ in $A / I P \cdot A$, we see that

$$
\pi\left(g_{i}\right)=\overline{b_{i}} \otimes n_{i}-\overline{c_{i-1}\left(v_{i}\right)} \otimes n_{i-1}-\cdots-\overline{c_{1}\left(v_{i}\right)} \otimes n_{1} .
$$

Recall that $A / I P \cdot A \otimes P \cong(\mathbb{Z} / d \mathbb{Z})^{k}$, where we let the $i$ th copy of $(\mathbb{Z} / d \mathbb{Z})^{k}$ be generated by the image of $\overline{1} \otimes n_{i}$ under the isomorphism. Then

$$
\pi\left(g_{i}\right)=(\overline{-c_{1}\left(v_{i}\right)}, \overline{-c_{2}\left(v_{i}\right)}, \ldots, \overline{-c_{i-1}\left(v_{i}\right)}, \overline{b_{i}}, \underbrace{0, \ldots, 0}_{k-i}),
$$

viewed as an element of $(\mathbb{Z} / d \mathbb{Z})^{k}$. Let $q_{i}=\left(-c_{1}\left(v_{i}\right), \ldots,-c_{i-1}\left(v_{i}\right), b_{i}, 0, \ldots, 0\right)$ in $\mathbb{Z}^{k}$. Let $x=\left(x_{1}, \ldots, x_{k}\right)$ be any element of $\mathbb{Z}^{k}$ such that $x_{1} r_{1}+\cdots+x_{k} r_{k}=$ $d$. Then the $q_{i}$ together with $x$ generate $\mathbb{Z}^{k}$, which implies that the $\pi\left(g_{i}\right)$ generate a subgroup of $(\mathbb{Z} / d \mathbb{Z})^{k}$ isomorphic to $(\mathbb{Z} / d \mathbb{Z})^{k-1}$.

\section{APPENDIX}

The purpose of this Appendix is to present some results of Robert Bieri and Ralph Strebel which we use in this paper. These results appeared in an unpublished set of notes entitled On groups of PL-homeomorphisms of the real line. First, recall that given a multiplicative subgroup $P$ and a $\mathbb{Z} P$-module $A$, then $I P$ is the augmentation ideal of the group ring $\mathbb{Z P}$ and $I P \cdot A$ is the submodule of $A$ generated by elements of the form $(1-p) a$, where $a \in A$ and $p \in P$.

Theorem A.1. Let $a, c, a^{\prime}$, and $c^{\prime}$ be elements of $A$ with $a<c$ and $a^{\prime}<c^{\prime}$. Then $\exists f$, a piecewise linear homeomorphism of $\mathbf{R}$, with slopes in $P$ and finitely many singularities, all in $A$, mapping $[a, c]$ onto $\left[a^{\prime}, c^{\prime}\right] \Leftrightarrow c^{\prime}-a^{\prime}$ is congruent to $c-$ a modulo $I P \cdot A$.

Proof of A.1. Assume first that such an $f$ exists. Let $a=b_{0}, b_{1}, \ldots, b_{n-1}$, $b_{n}=c$ be an increasing sequence of elements of $A$ such that $f$ is linear on $\left[b_{i-1}, b_{i}\right]$ with slope $p_{i}$, for all $i$. Then $c^{\prime}-a^{\prime}=\sum_{i=1}^{n} p_{i}\left(b_{i}-b_{i-1}\right)$. But $c-a=\sum_{i=1}^{n}\left(b_{i}-b_{i-1}\right)$, so $\left(c^{\prime}-a^{\prime}\right)-(c-a) \in I P \cdot A$.

Conversely, suppose there exist $a_{1}, \ldots, a_{n} \in A$ and $p_{1}, \ldots, p_{n} \in P$ such that

$$
\left(c^{\prime}-a^{\prime}\right)=(c-a)+\sum_{i-1}^{n}\left(1-p_{i}\right) a_{i}
$$

Set $b^{\prime}=c^{\prime}-a^{\prime}$ and $b=c-a$. If we could find $f$ mapping $[0, b]$ to $\left[0, b^{\prime}\right]$, composing $f$ with translations by $-c$ on the right and $c^{\prime}$ on the left gives the desired map. Now there exists a permutation $\pi$ of $\{1,2, \ldots, n\}$ such that the partial sums $b_{j}=b+\sum_{i=1}^{j}\left(1-p_{\pi(i)}\right) a_{\pi(i)}$ are positive for $j=0,1, \ldots, n$. If $f_{1}, f_{2}, \ldots, f_{n}$ are piecewise linear homeomorphisms of $\mathbf{R}$ with slopes in $P$ and singularities in $A$ such that $f_{j}\left(\left[0, b_{j-1}\right]\right)=\left[0, b_{j}\right]$, then $f_{n} \circ \cdots \circ f_{1}$ is the desired $f$. Therefore it suffices to prove the claim for $n=1$. Moreover, as $\left(1-p_{1}\right) a=\left(1-p_{1}^{-1}\right)\left(-p_{1}^{-1} a\right)$, we may assume that $p_{1}>1$. So we need to construct $f$ mapping $[0, b]$ to $[0, b+(p-1) a]$, where $p>1, a \neq 0$ (or the identity works), and $b$ and $b+(p-1) a$ are both positive. 
Suppose first that $a>0$. Choose a natural number $k$ with $a<p^{k} b$, and set $a^{\prime}=p^{-k} a$. Define $f_{1}, f_{2}: \mathbf{R} \rightarrow \mathbf{R}$ by

$$
\begin{aligned}
& f_{1}(t)= \begin{cases}t & \text { if } t \leq b-a^{\prime}, \\
p\left(t-\left(b-a^{\prime}\right)\right)+\left(b-a^{\prime}\right) & \text { if } b-a^{\prime}<t \leq b, \\
t+(p-1) a^{\prime} & \text { if } b<t,\end{cases} \\
& f_{2}(t)= \begin{cases}t & \text { if } t \leq b, \\
p^{k}(t-b)+b & \text { if } b<t \leq b+(p-1) a^{\prime}, \\
t+(p-1)\left(a-a^{\prime}\right) & \text { if } b+(p-1) a^{\prime}<t .\end{cases}
\end{aligned}
$$

Then $f_{1}$ maps $[0, b]$ to $\left[0, b+(p-1) a^{\prime}\right]$ and $f_{2}$ maps $\left[0, b+(p-1) a^{\prime}\right]$ onto $[0, b+(p-1) a]$, so $f=f_{2} \circ f_{1}$ maps $[0, b]$ to $[0, b+(p-1) a]$, and clearly has slopes and singularities as required.

On the other hand, if $a<0$ then there exists $f$ taking $[0, b+(p-1) a]$ to $[0,(b+(p-1) a)+(p-1)(-a)]=[0, b]$, so $f^{-1}$ will be the map we need.

Definition A.2. Let $P$ be a multiplicative subgroup with a finite basis $B$ consisting of integers. Let $l \in \mathbb{Z}$. We define a $P$-regular subdivision of level 1 of $[0, l]$ to be a sequence of the form $(0,1, \ldots, i, i+\Delta, i+2 \Delta, \ldots, i+$ $(n-1) \Delta, i, i+1, \ldots, l)$, where $n \in B, \Delta=\frac{1}{n}$, and $i=0,1, \ldots, l-1$. If $S^{\prime}=\left(0=b_{0}, b_{1}, \ldots, b_{h}=l\right)$ is a $P$-regular subdivision of level $k-1$, each sequence $S$ obtained from $S^{\prime}$ by replacing a pair $\left(b_{j}, b_{j+1}\right)$ of adjacent points by a sequence $\left(b_{j}, b_{j}+\Delta, b_{j}+2 \Delta, \ldots, b_{j+1}-\Delta, b_{j+1}\right)$ with $\Delta=\left(b_{j+1}-b_{j}\right) / n$ and $n \in B$, is by definition a $P$-regular subdivision of level $k$.

Notice that the definition depends on the choice of a basis for $P$ as well as on $P$ itself. We think of $P$ as being given with a specific basis. If we are given two $P$-standard subdivisions of the same size, $S$ and $S^{\prime}$, we can associate to them a piecewise linear homeomorphism of $[0, l]$ which takes as intervals the points of the first subdivision to the points of the second, and is linear on the intervals between subdivision points. We call this the affine interpolation of $S \times S^{\prime}$. Then we have:

Theorem A.3. Let $S=\left(0=b_{0}, b_{1}, \ldots, b_{h}=l\right)$ and $S^{\prime}=\left(0=b_{0}^{\prime}, b_{1}^{\prime}, \ldots, b_{h}^{\prime}\right.$ $=l)$ be P-regular subdivisions of $[0, l]$ with the same number of points. Then the affine interpolation of $S \times S^{\prime}$ belongs to $F(l, \mathbb{Z}[P], P)$. Conversely, if $f$ is a homeomorphism in $F$ it is the affine interpolation of two P-regular subdivisions of $[0, l]$.

Proof of A.3. If $S$ and $S^{\prime}$ are $P$-regular subdivisions the intervals $\left[b_{i}, b_{i+1}\right]$ and $\left[b_{i}^{\prime}, b_{i+1}^{\prime}\right]$ have as lengths elements of $P$, so each $b_{i}$ is in $\mathbb{Z}[P]$ aild the slopes of the affine interpolation are all in $P$, so it is in $F$.

Conversely, if $f$ is in $F$, we first construct a $P$-regular subdivision containing all the singularities of $f$. Let $d_{0}$ be a common denominator of all the singularities of $f$, and let $p_{0}$ be a multiple of $d_{0}$ which is a product of elements of $B$. Let $S$ be the subdivision of $[0, l]$ with $l p_{0}+1$ equidistant points. Then $S$ is $P$-regular. Next let

$$
f(S)=\left(0=f\left(b_{0}\right), f\left(b_{1}\right), \ldots, f\left(b_{h}\right)=l\right)
$$

be the $f$-image of $S$. Clearly $f$ is the affine interpolation of $S$ and $f(S)$, but $f(S)$ is not necessarily $P$-regular. Say $f$ has slope $s_{i}$ on $\left[b_{i}, b_{i+1}\right]$. Then 
$f\left(b_{i+1}\right)-f\left(b_{i}\right)=s_{i}\left(b_{i+1}-b_{i}\right)$ which is in $P$. Now let $S^{\prime}$ be an equidistant $P$-regular subdivision containing all the points of $f(S)$; let $\Delta=\frac{1}{p}$ be the step width of $S^{\prime}$. Then the interval $\left[f\left(b_{i}\right), f\left(b_{i+1}\right)\right]$ is divided into an integral number $m_{i}$ of subintervals of $S^{\prime}$. On the other hand, $m_{i}=p s_{i}\left(b_{i+1}-b_{i}\right)$ and so is in $P$. It follows that $\exists q_{i}$, a product of elements of $B$, such that $q_{i} m_{i}$ is a product of elements of $B$. Subdivide each subinterval of step width $\Delta$ of $\left[f\left(b_{i}\right), f\left(b_{i+1}\right)\right]$ into $q_{i}$ equidistant steps. If this is done for all $i$ one ends up with a $P$-regular subdivision $S^{\prime \prime}$ having the property that its inverse image $f^{-1}\left(S^{\prime \prime}\right)$ is also $P$-regular. As $f$ is the affine interpolation $f^{-1}\left(S^{\prime \prime}\right) \times S^{\prime \prime}$ the theorem is proved.

Theorem A.4. Let $B(l, A, P)$ be the subgroup of $F(l, A, P)$ consisting of homeomorphisms which are the identity in neighborhoods of 0 and $l$. Let $B(\mathbf{R}, A, P)$ be the group of piecewise linear homeomorphisms of the real line with finitely many singularities, all in $A$, and slopes in $P$, which are the identity in neighborhoods of $+\infty$ and $-\infty$. Then $B(l, A, P) \cong B(\mathbf{R}, A, P)$.

Proof of A.4. Choose $a_{0}>0, a_{0} \in A$. Since $P$ contains arbitrarily small positive elements, we can choose a doubly infinite increasing sequence $\left\{b_{i} \mid i \in\right.$ $\mathbb{Z}\}$ of elements in $A \cap(0, l)$ such that:

(i) $b_{i+1}-b_{i} \in P \cdot a_{0} \quad \forall i \in \mathbb{Z}$,

(ii) $\lim _{i \rightarrow-\infty} b_{i}=0$ and $\lim _{i \rightarrow+\infty} b_{i}=l$.

Let $\varphi: \mathbf{R} \rightarrow(0, l)$ be the piecewise linear map taking $\left(i a_{0},(i+1) a_{0}\right)$ linearly to $\left(b_{i}, b_{i+1}\right)$, for all $i \in \mathbb{Z}$. Since elements of $B(l, A, P)$ move at most finitely many of the $b_{i}$, and elements of $B(\mathbf{R}, A, P)$ move at most finitely many of the $i a_{0}$, conjugation by $\varphi$ induces an isomorphism between $B(\mathbf{R}, A, P)$ and $B(l, A, P)$.

The final result from the notes of Bieri and Strebel is the observation that the commutator subgroup of $B(l, A, P)$ is simple. The authors apply the following restatement of a theorem of G. Higman [10].

Theorem A.5. Let $\Omega$ be a totally ordered set and let $B$ be a two-fold transitive, ordered permutation group on $\Omega$ consisting of bounded elements. Then $[B, B]$ is a simple group $\neq\{1\}$.

Here, two-fold transitive just means that if $a<a^{\prime}$ and $b<b^{\prime}$, with $a, a^{\prime}, b$, and $b^{\prime} \in \Omega$, then there is an element of $B$ taking $a$ to $b$ and $a^{\prime}$ to $b^{\prime}$. This result can be applied with $B=B(l, A, P)$ and $\Omega=I P \cdot A \cap(0, l)$. We know from A.1 that $F(l, A, P)$ is two-fold transitive on $\Omega$, and since $\Omega$ is dense in $(0, l)$ it follows that $B$ is also two-fold transitive on $\Omega$. Hence $[B, B]$ is simple.

\section{REFERENCES}

1. K. S. Brown and R. Geoghegan, An infinite-dimensional torsion-free $F P_{\infty}$ group, Invent. Math. 77 (1984), 367-381.

2. K. S. Brown, Finiteness properties of groups, J. Pure Appl. Algebra 44 (1987), 45-75.

3. __ The geometry of rewriting systems: A proof of the Anick-Groves-Squier theorem, Algorithms and Classification in Combinatorial Group Theory, edited by G. Baumslag and C. F. Miller, MSRI publ. 23, Springer-Verlag, 1992, pp. 137-163. 
4. _ Presentations for groups acting on simply connected complexes, J. Pure Appl. Algebra 32 (1984), 1-10.

5. R. Brown, Elements of modern topology, McGraw-Hill, 1968.

6. G. Cooke and R. Finney, Homology of cell complexes, Princeton Univ. Press, 1967.

7. D. B. A. Epstein, The simplicity of certain groups of homeomorphisms, Compositio Math. 22 (1970), 165-173.

8. P. Greenberg, Pseudogroups from group actions, Amer. J. Math. 109 (1987), 893-906.

9. G. Higman, Finitely presented infinite simple groups, Notes on Pure Math., vol. 8, Australian National University, Canberra, 1974.

10. __ On infinite simple permutation groups, Publ. Math. Debrecen 3 (1954), 221-226.

11. A. T. Lundell and S. Weingram, The topology of $C W$ complexes, Van Nostrand Reinhold, New York, 1969.

12. E. A. Scott, A construction which can be used to produce finitely presented simple groups, J. Algebra 90 (1984), 294-322.

13. $\ldots$ The embedding of certain linear and abelian groups in finitely presented infinite simple groups, J. Algebra 90 (1984), 323-332.

14. __ A finitely presented simple group with unsolvable conjugacy problem, J. Algebra 90 (1984), 333-353.

15. D. Quillen, Homotopy properties of the poset of nontrivial p-subgroups of a group, Adv. in Math. 28 (1978), 101-128.

Department of Mathematics, White Hall, Cornell University, Ithaca, New York 14853

Current address: Department of Mathematics, Ohio State University, Columbus, Ohio 43210

E-mail address: melanie@function.mps.ohio-state.edu 